TRANSACTIONS OF THE

AMERICAN MATHEMATICAL SOCIETY

Volume 360, Number 5, May 2008, Pages 2303-2325

S 0002-9947(07)04308-5

Article electronically published on December 11, 2007

\title{
OPTIMAL CONSTANTS IN THE EXCEPTIONAL CASE OF SOBOLEV INEQUALITIES ON RIEMANNIAN MANIFOLDS
}

\author{
ZOÉ FAGET
}

\begin{abstract}
Let $(M, g)$ be a Riemannian compact $n$-manifold. We know that for any $\varepsilon>0$, there exists $C_{\varepsilon}>0$ such that for any $u \in H_{1}^{n}(M), \int_{M} e^{u} \mathrm{dv}_{g} \leq$ $C_{\varepsilon} \exp \left[\left(\mu_{n}+\varepsilon\right) \int_{M}|\nabla u|^{n} \mathrm{dv}_{g}+\frac{1}{\operatorname{vol}(M)} \int_{M} u \mathrm{dv}_{g}\right], \mu_{n}$ being the smallest constant possible such that the inequality remains true for any $u \in H_{1}^{n}(M)$. We call $\mu_{n}$ the "first best constant". We prove in this paper that it is possible to choose $\varepsilon=0$ and keep $C_{\varepsilon}$ a finite constant. In other words we prove the existence of a "second best constant" in the exceptional case of Sobolev inequalities on compact Riemannian manifolds.
\end{abstract}

\section{INTRODUCTION}

Let $(M, g)$ be a smooth compact Riemannian $n$-manifold, $n \geq 2$. For $1 \leq p \leq n$, we let $H_{1}^{p}(M)$ be the Sobolev space of order $p$, that is, the completion of $\mathcal{C}^{\infty}(M)$ with respect to the norm

$$
\|u\|_{H_{1}^{p}}^{p}=\|\nabla u\|_{p}^{p}+\|u\|_{p}^{p}
$$

where $\|.\|_{p}$ stands for the $L^{p}$ norm $\|u\|_{p}=\left(\int_{M}|u|^{p} \mathrm{dv}_{g}\right)^{1 / p}$. It is well known that the critical Sobolev embedding $H_{1}^{p}(M) \subset L^{p^{*}}(M)$, where $1 \leq p<n, p^{*}=\frac{n p}{n-p}$, is continuous, which gives the existence of two constants $A$ and $B$ such that for any $u \in H_{1}^{p}(M)$

$$
\left(I_{p}\right) \quad\|u\|_{p^{*}}^{p} \leq A\|\nabla u\|_{p}^{p}+B\|u\|_{p}^{p} \text {. }
$$

The study of best constants in this inequality amounts to detecting the smallest $A$ and $B$ such that the inequality holds for any $u \in H_{1}^{p}(M)$. Priority is given to the former constant $A$ because of applications, in particular applications to prescribed scalar curvature problems. The first question is: what is the precise value of the former best constant, namely, what is the value of $\alpha_{p}=\inf \mathcal{A}_{p}$, where

$$
\mathcal{A}_{p}=\left\{A \in \mathbb{R} \mid \exists B \in \mathbb{R} \text { such that }\left(I_{p}\right) \text { is valid } \forall u \in H_{1}^{p}(M)\right\} ?
$$

The next questions are: is $\mathcal{A}_{p}$ a closed set (in other words, is $\alpha_{p}$ reached); can one compute or estimate the latter best constant (the smallest $B$ possible with $A=\alpha_{p}$ ); and finally, under which conditions do functions exist that render $\left(I_{p}\right)$ an equality? All these questions as well as applications have been widely studied, and complete or partial answers have been given. We refer, among many others, to the works of

Received by the editors December 8, 2005.

2000 Mathematics Subject Classification. Primary $46 \mathrm{E} 35$.

Key words and phrases. Best constants, optimal Sobolev inequalities, exceptional case, concentration phenomenon. 
Aubin, Bandle, Djadli, Druet, Hebey, Vaugon [1], [3] [5, 6] [9], [10], [11], [12] [13], [15], [16], [17], [18].

In the borderline case where $p=n, H_{1}^{n}(M)$ is not embedded in $L^{\infty}(M)$. However, $H_{1}^{n}(M) \subset L^{q}(M)$ for any $q \geq 1$, and $e^{u} \in L^{1}(M)$ for any $u \in H_{1}^{n}(M)$, which gives the existence of two constants $\mu$ and $C$ such that

$$
\int_{M} e^{u} \mathrm{dv}_{g} \leq C \exp \left[\mu\|\nabla u\|_{n}^{n}\right]
$$

for any $u \in H_{1}^{n}(M)$ satisfying $\int_{M} u \mathrm{dv}_{g}=0$. When considering this new problem, which we call the exceptional case, we can ask once again the same questions regarding the best constants, this time giving priority to $\mu$. In contrast to the classical case, the study of the exceptional case hasn't gone further than the study of the first best constant. Cherrier [8] proved that for any $\varepsilon>0$, there exists $A_{\varepsilon}$, $B_{\varepsilon}$ such that for any $u \in H_{1}^{n}(M)$

$$
\int_{M} e^{u} \mathrm{dv}_{g} \leq B_{\varepsilon} \exp \left[\left(\mu_{n}+\varepsilon\right)\|\nabla u\|_{n}^{n}+A_{\varepsilon}\|u\|_{n}^{n}\right]
$$

and that $\mu_{n}=(n-1)^{n-1} n^{1-2 n} w_{n-1}^{-1}$ is the smallest possible $\mu$ such that the inequality remains true for any $u \in H_{1}^{n}(M)$. Aubin [2] proved that for any $\varepsilon>0$, there exists $C_{\varepsilon}$ such that

$$
\int_{M} e^{u} \mathrm{dv}_{g} \leq C_{\varepsilon} \exp \left[\left(\mu_{n}+\varepsilon\right)\|\nabla u\|_{n}^{n}\right]
$$

for any $u \in H_{1}^{n}(M)$ satisfying $\int_{M} u \mathrm{dv}_{g}=0$, with the same $\mu_{n}$ as above, and $\mu_{n}$ is the smallest constant possible such that the inequality holds for any $u \in H_{1}^{n}(M)$ with $\int_{M} u \mathrm{dv}_{g}=0$. In fact, (2) implies a stronger version of (1): for any $\varepsilon, \varepsilon^{\prime}$, there exists $C_{\varepsilon, \varepsilon^{\prime}}>0$ such that for any $u \in H_{1}^{n}(M)$

$$
\int_{M} e^{u} \operatorname{dv}_{g} \leq C_{\varepsilon, \varepsilon^{\prime}} \exp \left[\left(\mu_{n}+\varepsilon\right)\|\nabla u\|_{n}^{n}+\varepsilon^{\prime}\|u\|_{n}^{n}\right] .
$$

The precise value of the first best constant in the exceptional case of Sobolev inequalities appears directly in results on the existence of nontrivial solutions of nonlinear PDE's of the following type:

$$
\Delta_{n} u+a=f e^{u},
$$

where $\Delta_{n} u=-\operatorname{div}\left(|\nabla u|^{n-2} \nabla u\right), a \in \mathbb{R}$ and $f$ is a smooth function.

Until now, the question regarding the existence of a second best constant (precisely, is it possible to take $\varepsilon=0$ in (1) or (2) and still have a finite $A_{\varepsilon}, B_{\varepsilon}$ or $C_{\varepsilon}$ ), has only been studied in very specific cases. Cherrier [8] proved that when $n=2$, $\mu_{2}$ is reached in (1). Aubin [2] proved that on the sphere $S_{n}$ the first best constant is reached. Recently, the author gave an answer for compact locally conformaly flat manifolds [15]. In this paper, we give a complete answer to this question.

We prove the theorem

Theorem. Let $(M, g)$ be a compact Riemannian n-manifold. There exists $C>0$ such that for any $u \in H_{1}^{n}(M)$ satisfying $\int_{M} u \mathrm{dv}_{g}=0$

$$
\int_{M} e^{u} \mathrm{dv}_{g} \leq C \exp \left[\mu_{n} \int_{M}|\nabla u|^{n} \mathrm{dv}_{g}\right],
$$


where $\mu_{n}=(n-1)^{n-1} n^{1-2 n} w_{n-1}^{-1}, w_{n-1}$ is the volume of the standard unit sphere $S_{n-1}$. Furthermore, $\mu_{n}$ is the smallest constant such that the above inequality remains true for any $u \in H_{1}^{n}$ satisfying $\int_{M} u \mathrm{dv}_{g}=0$.

In order to prove the theorem, we need to prove a weaker result first.

Proposition. Let $(M, g)$ be a compact Riemannian $n$-manifold. For any $\varepsilon>0$, there exists $C_{\varepsilon}>0$ such that for any $u \in H_{1}^{n}(M)$

$$
\int_{M} e^{u} \mathrm{dv}_{g} \leq C_{\varepsilon} \exp \left[\mu_{n} \int_{M}|\nabla u|^{n} \mathrm{dv}_{g}+\varepsilon \int_{M}|u|^{n} \mathrm{dv}_{g}\right],
$$

where $\mu_{n}=(n-1)^{n-1} n^{1-2 n} w_{n-1}^{-1}, w_{n-1}$ is the volume of the standard unit sphere $S_{n-1}$. Furthermore, $\mu_{n}$ is the smallest constant such that the above inequality remains true for any $u \in H_{1}^{n}(M)$.

1.1. A word on the proofs of this paper: concentration phenomena applied to the study of optimal constants. The proof of the proposition is by contradiction: if the best constant is not reached, we can build a family of functions $\left(u_{\alpha}\right)_{\alpha>0}$ that verify what we call a concentration phenomenon. Examples of concentration phenomena applied to optimal constants problems can be found in [10], [12], [14]. The study of this concentration phenomenon leads to the desired contradiction.

The idea is that functions concentrating around a point could be seen as functions with a compact support on a Euclidean ball. However, they do not have a compact support indeed, and the ball is not Euclidean! The first difficulty is to prove that outside of a concentration ball, the functions converge well enough to zero. It is even more difficult to overcome the fact that the ball is not Euclidean, and this is where the main problems are. To get rid of the "perturbations" brought by the metric, we have to prove a fundamental inequality on the $u_{\alpha}$.

We divide the proof into several steps. The convergence outside the concentration ball is studied in Steps 1 to 3. The proof of the fundamental inequality that we need will take Steps 4 to 6 , and will use a powerful tool: a blow up argument to watch precisely the behaviour of the functions $u_{\alpha}$. Steps 5 and 6 are dedicated to proofs of subtle bounds on the functions. This part is where all the main new technical as well as conceptual ideas appear, in regard to the classical case. The rest of the proof rests upon the results we have on $\mathbb{R}^{n}$.

The proof of the main Theorem uses the result established in the Proposition.

1.2. Notations and preliminary results. Throughout this article, $(M, g)$ is a compact Riemannian manifold of dimension $n$.

We denote for any $p, q \geq 1 H_{1}^{p}(M)=H_{1}^{p}, L^{q}(M)=L^{q}(q$ possibly equals infinity).

We recall some standard results. For any $p \geq 1$ we have the compact embedding $H_{1}^{n} \subset L^{p}$, which gives a "classical" Sobolev inequality: for any $p>q \geq 1$, for any $\varepsilon>0$, there exists $C_{\varepsilon}>0$ such that for any $u \in H_{1}^{n}$

$$
\left(\int_{M} u^{p} \mathrm{dv}_{g}\right)^{n / p} \leq \varepsilon \int_{M}|\nabla u|^{n} \mathrm{dv}_{g}+C_{\varepsilon}\left(\int_{M}|u|^{q} \mathrm{dv}_{g}\right)^{n / q} .
$$

As stated in the introduction, the application $H_{1}^{n} \ni u \rightarrow e^{u} \in L^{1}$ is compact. The "exceptional" Sobolev inequalities (2) and (3) are associated to this embedding. 
In the case of a Euclidean ball $B$, we know from [8] that it is possible to take $\varepsilon=0$ and keep $C_{\varepsilon}>0$ finite, i.e. for any $u \in H_{1}^{n}$

$$
\int_{B} e^{u} \mathrm{~d} \xi \leq C \exp \left[\mu_{n} \int_{B}|\nabla u|^{n} \mathrm{~d} \xi\right]
$$

where $\mathrm{d} \xi$ is the Euclidean element of volume.

For short we set, for any $u \in H_{1}^{n}$

$$
\Delta_{n} u=\Delta_{n, g} u=-\operatorname{div}\left(|\nabla u|^{n-2} \nabla u\right),
$$

and for any $u, v \in H_{1}^{n}, \nabla u \nabla v$ denotes the scalar product of the gradients.

\section{Proof of the Proposition}

Proof. We proceed by contradiction. Assume that there exists $\tilde{\varepsilon}>0$ such that for any $\alpha>0$ there exists $v_{\alpha} \in H_{1}^{n}$ satisfying

$$
\ln \left(\int_{M} e^{v_{\alpha}} \mathrm{dv}_{g}\right)>\alpha+\mu_{n} \int_{M}\left|\nabla v_{\alpha}\right|^{n} \mathrm{dv}_{g}+\tilde{\varepsilon} \int_{M}\left|v_{\alpha}\right|^{n} \mathrm{dv}_{g} .
$$

For any $\alpha>0$ and $u \in H_{1}^{n}$, we define

$$
I_{\alpha}(u)=\frac{\mu_{n} \int_{M}|\nabla u|^{n} \mathrm{dv}_{g}+\tilde{\varepsilon} \int_{M}|u|^{n} \mathrm{dv}_{g}+\alpha}{\ln \left(\int_{M} e^{u} \mathrm{dv}_{g}\right)} .
$$

Let $\lambda_{\alpha}=\inf _{u \in H} I_{\alpha}(u)$, where $H=\left\{u \in H_{1}^{n} \mid \int_{M} e^{u} \mathrm{dv}_{g}>1\right\}$. We have $0<\lambda_{\alpha}<1$. By standard variational techniques the infimum is attained and the minimizer will be denoted by $u_{\alpha}$. Since $I_{\alpha}(|u|) \leq I_{\alpha}(u)$ we can choose $u_{\alpha} \geq 0$.

We have $I_{\alpha}\left(u_{\alpha}\right)=\lambda_{\alpha}$, i.e.

$$
\mu_{n} \int_{M}\left|\nabla u_{\alpha}\right|^{n} \operatorname{dv}_{g}+\tilde{\varepsilon} \int_{M} u_{\alpha}^{n} \operatorname{dv}_{g}+\alpha=\lambda_{\alpha} \ln \int_{M} e^{u_{\alpha}} \mathrm{dv}_{g}
$$

Since $u_{\alpha}$ is a minimizer for $I_{\alpha}, I_{\alpha}^{\prime}(\Phi)=0$ and $I_{\alpha}^{\prime \prime}(\Phi, \Phi) \geq 0$ for any $\Phi \in H_{1}^{n}$. In other words

$$
n \mu_{n} \int_{M}\left|\nabla u_{\alpha}\right|^{n-2} \nabla u_{\alpha} \nabla \Phi \operatorname{dv}_{g}+\tilde{\varepsilon} n \int_{M} u_{\alpha}^{n-1} \Phi \operatorname{dv}_{g}=\lambda_{\alpha} \frac{\int_{M} e^{u_{\alpha}} \Phi \mathrm{dv}_{g}}{\int_{M} e^{u_{\alpha}} \operatorname{dv}_{g}}
$$

and

$$
\begin{array}{r}
\lambda_{\alpha} \frac{\int_{M} e^{u_{\alpha}} \Phi^{2} \mathrm{dv}_{g}}{\int_{M} e^{u_{\alpha}} \mathrm{dv}_{g}} \leq \mu_{n} n(n-1) \int_{M}\left|\nabla u_{\alpha}\right|^{n-2}|\nabla \Phi|^{2} \mathrm{dv}_{g} \\
\quad+\tilde{\varepsilon} n(n-1) \int_{M} u_{\alpha}^{n-2} \Phi^{2} \mathrm{dv}_{g}+\lambda_{\alpha}\left(\frac{\int_{M} e^{u_{\alpha}} \Phi \mathrm{dv}_{g}}{\int_{M} e^{u_{\alpha}} \mathrm{dv}_{g}}\right) .
\end{array}
$$

Furthermore, by [21], $u_{\alpha} \in \mathcal{C}^{1, \beta}$ and $u_{\alpha} \in \mathcal{C}^{\infty}$ if $n=2$.

For simplicity, all positive constants independent of $\alpha$ will be denoted $C$.

Step 1. We first prove some results for the functions $u_{\alpha}$. When $\alpha \rightarrow+\infty$, we immediately get from (6) that

$$
\lim _{\alpha \rightarrow+\infty} \int_{M} e^{u_{\alpha}} \mathrm{dv}_{g}=+\infty
$$

We claim that, for any $q \geq 1$, there exists $C$ such that

$$
\int_{M} u_{\alpha}^{q} \mathrm{dv}_{g} \leq C
$$


Proof. Testing $\Phi=1$ in (7) gives $\int_{M} u_{\alpha}^{n-1} \mathrm{dv}_{g} \leq C$. Since

$$
\left|\nabla u_{\alpha}\right|^{n-2} \nabla u_{\alpha} \nabla\left(u_{\alpha}+1\right)^{-1 / 2}=-C\left|\nabla\left(u_{\alpha}+1\right)^{\frac{2 n-3}{2 n}}\right|^{n},
$$

testing $\Phi=\left(u_{\alpha}+1\right)^{1 / 2}$ in (7) subsequently gives

$$
\begin{aligned}
\int_{M} \mid & \left.\nabla\left(u_{\alpha}+1\right)^{\frac{2 n-3}{2 n}}\right|^{n} \mathrm{dv}_{g} \\
& \leq C \int_{M} u_{\alpha}^{n-1}\left(u_{\alpha}+1\right)^{-1 / 2} \mathrm{dv}_{g}-C \frac{\int_{M} e^{u_{\alpha}}\left(u_{\alpha}+1\right)^{-1 / 2} \mathrm{dv}_{g}}{\int_{M} e^{u_{\alpha}} \mathrm{dv}_{g}} \\
& \leq C .
\end{aligned}
$$

Inequalities (4) and (11) give

$$
\int_{M}\left(u_{\alpha}+1\right)^{\frac{(2 n-3) p}{2 n}} \mathrm{dv}_{g} \leq C
$$

for any $p \geq 1$. Inequality (10) follows.

We now claim that for any $p<n$, there exists $C$ such that

$$
\int_{M}\left|\nabla u_{\alpha}\right|^{p} \mathrm{dv}_{g} \leq C
$$

Proof. We have

$$
\int_{M}\left|\nabla u_{\alpha}\right|^{p} \mathrm{dv}_{g}=C \int_{M}\left(u_{\alpha}+1\right)^{3 p / 2 n}\left|\nabla\left(u_{\alpha}+1\right)^{\frac{2 n-3}{2 n}}\right|^{p} \mathrm{dv}_{g} .
$$

Hence Hölder inequality and inequalities (10) and (11) give (12).

We now prove two results in a general form.

Let $\Omega$ be an open subset of $M$ such that

$$
\varlimsup_{\alpha \rightarrow+\infty} \frac{\int_{\Omega} e^{u_{\alpha}} \mathrm{dv}_{g}}{\int_{M} e^{u_{\alpha}} \mathrm{dv}_{g}}=k<1 .
$$

Then

(1) For any open subset $\Omega^{\prime}$ of $\Omega$ such that $\overline{\Omega^{\prime}} \subset \Omega$, there exists $C_{1}=C_{1}\left(k, \Omega, \Omega^{\prime}\right)$ such that

$$
\int_{\Omega^{\prime}}\left|\nabla u_{\alpha}\right|^{n} \operatorname{dv}_{g} \leq C_{1} .
$$

(2) For any open subset $\Omega^{\prime}$ of $\Omega$ such that $\overline{\Omega^{\prime}} \subset \Omega$, there exists $C_{2}=C_{2}\left(k, \Omega, \Omega^{\prime}, p\right)$ such that

$$
\int_{\Omega^{\prime}} e^{p u_{\alpha}} \mathrm{dv}_{g} \leq C_{2}
$$

for any $p>0$.

In order to prove (14) we test $\Phi=\eta^{n / 2}\left(u_{\alpha}+a\right)^{1 / 2}$ in (8), where $a \in \mathbb{R}, a>0$ and $\eta$ is a $\mathcal{C}^{\infty}$ cut off function of $\Omega^{\prime}$, i.e. $\eta=1$ on $\Omega^{\prime}, \eta=0$ on $M \backslash \Omega$ and $0 \leq \eta \leq 1$. We get

$$
\begin{array}{r}
\lambda_{\alpha} \frac{\int_{M} \eta^{n} e^{u_{\alpha}}\left(u_{\alpha}+a\right) \mathrm{dv}_{g}}{\int_{M} e^{u_{\alpha}} \mathrm{dv}_{g}} \leq \mu_{n} n(n-1) \int_{M}\left|\nabla u_{\alpha}\right|^{n-2}\left|\nabla\left(\eta^{n / 2}\left(u_{\alpha}+a\right)^{1 / 2}\right)\right|^{2} \mathrm{dv}_{g} \\
+\tilde{\varepsilon} n(n-1) \int_{M} u_{\alpha}^{n-2} \eta^{n}\left(u_{\alpha}+a\right) \mathrm{dv}_{g}+\lambda_{\alpha}\left(\frac{\int_{M} e^{u_{\alpha}} \eta^{n / 2}\left(u_{\alpha}+a\right)^{1 / 2} \mathrm{dv}_{g}}{\int_{M} e^{u_{\alpha}} \mathrm{dv}_{g}}\right)^{2}
\end{array}
$$


By Hölder's inequality and assumption (13)

$$
\left(\frac{\int_{M} e^{u_{\alpha}} \eta^{n / 2}\left(u_{\alpha}+a\right)^{1 / 2} \mathrm{dv}_{g}}{\int_{M} e^{u_{\alpha}} \mathrm{dv}_{g}}\right)^{2} \leq k \frac{\int_{M} e^{u_{\alpha}} \eta^{n}\left(u_{\alpha}+a\right) \mathrm{dv}_{g}}{\int_{M} e^{u_{\alpha}} \mathrm{dv}_{g}} .
$$

Hence,

$$
\begin{aligned}
\lambda_{\alpha}(1 & -k) \frac{\int_{M} \eta^{n} e^{u_{\alpha}}\left(u_{\alpha}+a\right) \mathrm{dv}_{g}}{\int_{M} e^{u_{\alpha}} \operatorname{dv}_{g}} \\
& \leq \mu_{n} n(n-1) \int_{M}\left|\nabla u_{\alpha}\right|^{n-2}\left|\nabla\left(\eta^{n / 2}\left(u_{\alpha}+a\right)^{1 / 2}\right)\right|^{2} \mathrm{dv}_{g} \\
& +\tilde{\varepsilon} n(n-1) \int_{M} u_{\alpha}^{n-2} \eta^{n}\left(u_{\alpha}+a\right) \operatorname{dv}_{g}
\end{aligned}
$$

where $1-k>0$. Furthermore

$$
\begin{aligned}
\int_{M}\left|\nabla u_{\alpha}\right|^{n-2} \mid \nabla\left(\eta^{n / 2}\left(u_{\alpha}+a\right)^{1 / 2}\right) & \left.\right|^{2} \mathrm{dv}_{g} \leq C \int_{M} \eta^{n}\left|\nabla u_{\alpha}\right|^{n}\left(u_{\alpha}+a\right)^{-1} \mathrm{dv}_{g} \\
& +C \int_{M}\left|\nabla u_{\alpha}\right|^{n-2} \eta^{n-2}\left(u_{\alpha}+a\right)|\nabla \eta|^{2} \mathrm{dv}_{g} .
\end{aligned}
$$

We bound the first term on the right hand side:

$$
\int_{M} \eta^{n}\left|\nabla u_{\alpha}\right|^{n}\left(u_{\alpha}+a\right)^{-1} \mathrm{dv}_{g} \leq \frac{C}{a} \int_{M}\left|\nabla u_{\alpha}\right|^{n} \eta^{n} \mathrm{dv}_{g}
$$

For the second term on the right hand side, we have that, for any $\varepsilon>0$

$$
\begin{aligned}
& \int_{M}\left|\nabla u_{\alpha}\right|^{n-2} \eta^{n-2}\left(u_{\alpha}+a\right)|\nabla \eta|^{2} \mathrm{dv}_{g} \\
& \quad \leq \varepsilon \int_{M}\left(\eta\left|\nabla u_{\alpha}\right|\right)^{n} \mathrm{dv}_{g}+C_{\varepsilon} \int_{M}\left(u_{\alpha}+a\right)^{n / 2}|\nabla \eta|^{n} \mathrm{dv}_{g} .
\end{aligned}
$$

Hence, combining (17), (18), (19) in (16)

$$
\begin{aligned}
\lambda_{\alpha}(1 & -k) \frac{\int_{M} e^{u_{\alpha}} \eta^{n}\left(u_{\alpha}+a\right) \mathrm{dv}_{g}}{\int_{M} e^{u_{\alpha}} \mathrm{dv}_{g}} \\
\leq & \left(\frac{C}{a}+\varepsilon\right) \int_{M}\left|\nabla u_{\alpha}\right|^{n} \eta^{n}+C_{\varepsilon} \int_{M}\left(u_{\alpha}+a\right)^{n / 2}|\nabla \eta|^{n} \mathrm{dv}_{g} \\
& +C \int_{M} u_{\alpha}^{n-2} \eta^{n}\left(u_{\alpha}+a\right) \mathrm{dv}_{g} .
\end{aligned}
$$

Since $\int_{M} u_{\alpha}^{p} \mathrm{dv}_{g} \leq C$ for any $p \geq 1$ by (10), we obtain

$$
\frac{\int_{M} e^{u_{\alpha}} \eta^{n}\left(u_{\alpha}+a\right) \mathrm{dv}_{g}}{\int_{M} e^{u_{\alpha}} \mathrm{dv}_{g}} \leq\left(\frac{C}{a}+\varepsilon\right) \int_{M}\left|\nabla u_{\alpha}\right|^{n} \eta^{n} \mathrm{dv}_{g}+C_{a} .
$$

Testing $\Phi=\eta^{n} u_{\alpha}$ in (7) we get

$$
n \mu_{n} \int_{M}\left|\nabla u_{\alpha}\right|^{n-2} \nabla u_{\alpha} \nabla\left(\eta^{n} u_{\alpha}\right) \mathrm{dv}_{g}+\tilde{\varepsilon} n \int_{M} \eta^{n} u_{\alpha}^{n} \mathrm{dv}_{g}=\lambda_{\alpha} \frac{\int_{M} e^{u_{\alpha}} \eta^{n} u_{\alpha} \mathrm{dv}_{g}}{\int_{M} e^{u_{\alpha}} \mathrm{dv}_{g}}
$$

which gives, keeping in mind that $\int_{M} \eta^{n} u_{\alpha}^{n} \mathrm{dv}_{g} \geq 0$,

$$
n \mu_{n} \int_{M}\left|\nabla u_{\alpha}\right|^{n-2} \nabla u_{\alpha} \nabla\left(\eta^{n} u_{\alpha}\right) \mathrm{dv}_{g} \leq \lambda_{\alpha} \frac{\int_{M} e^{u_{\alpha}} \eta^{n} u_{\alpha} \mathrm{dv}_{g}}{\int_{M} e^{u_{\alpha}} \mathrm{dv}_{g}} .
$$


For any $\varepsilon>0$, there exists a $C_{\varepsilon}>0$ such that

$$
\int_{M}\left|\nabla u_{\alpha}\right|^{n-2} \nabla u_{\alpha} \nabla\left(\eta^{n} u_{\alpha}\right) \mathrm{dv}_{g} \geq(1-\varepsilon) \int_{M}\left|\nabla u_{\alpha}\right|^{n} \eta^{n} \mathrm{dv}_{g}-C_{\varepsilon} \int_{M} u_{\alpha}^{n}|\nabla \eta|^{n} \mathrm{dv}_{g} .
$$

Therefore, by (20) and (21)

$$
(1-\varepsilon) \int_{M}\left|\nabla u_{\alpha}\right|^{n} \eta^{n} \mathrm{dv}_{g}-C_{\varepsilon} \int_{M} u_{\alpha}^{n}|\nabla \eta|^{n} \mathrm{dv}_{g} \leq\left(\frac{C}{a}+\varepsilon\right) \int_{M}\left|\nabla u_{\alpha}\right|^{n} \eta^{n} \mathrm{dv}_{g}+C_{a} .
$$

We choose $a$ and $\varepsilon$ such that $1-C / a-2 \varepsilon>0$, and by (10) and the construction of $\eta$, inequality (14) follows.

We now prove (15). We choose $\Omega^{\prime \prime} \subset \Omega$ such that $\int_{\Omega^{\prime \prime}}\left|\nabla u_{\alpha}\right|^{n} \mathrm{dv}_{g} \leq C$ (this is possible according to (14)) and $\bar{\Omega}^{\prime} \subset \Omega^{\prime \prime}$. By (3) we get, for a cut off function $\eta$ of $\Omega^{\prime}$, for any $p \geq 0$

$$
\begin{aligned}
\int_{\Omega^{\prime}} e^{p u_{\alpha}} \mathrm{dv}_{g} & \leq \int_{M} e^{p \eta u_{\alpha}} \mathrm{dv}_{g} \\
& \leq C \exp \left[C p^{n} \int_{M}\left|\nabla\left(\eta u_{\alpha}\right)\right|^{n} \mathrm{dv}_{g}+C p^{n} \int_{M}\left(\eta u_{\alpha}\right)^{n} \mathrm{dv}_{g}\right] \\
& \leq C \exp \left[C_{p} \int_{\Omega^{\prime}}\left|\nabla u_{\alpha}\right|^{n} \mathrm{dv}_{g}+C_{p} \int_{M} u_{\alpha}^{n} \mathrm{dv}_{g}\right] \\
& \leq C .
\end{aligned}
$$

Step 2. We start with a definition.

Definition (Concentration point). A point $x_{0}$ is called a concentration point of $\left(u_{\alpha}\right)_{\alpha>0}$ if for any $\delta>0$

$$
\varlimsup_{\alpha \rightarrow+\infty} \frac{\int_{B_{x_{0}}(\delta)} e^{u_{\alpha}} \mathrm{dv}_{g}}{\int_{M} e^{u_{\alpha}} \mathrm{dv}_{g}}>0 .
$$

Since $M$ is compact there exists at least one point of concentration of $\left(u_{\alpha}\right)_{\alpha>0}$. Let $x_{0}$ be a concentration point. We claim that

$$
\varlimsup_{\alpha \rightarrow+\infty} \frac{\int_{B_{x_{0}}(\delta)} e^{u_{\alpha}} \mathrm{dv}_{g}}{\int_{M} e^{u_{\alpha}} \mathrm{dv}_{g}}=1 .
$$

Proof. We proceed by contradiction: assume that

$$
\varlimsup_{\alpha \rightarrow+\infty} \frac{\int_{B_{x_{0}}(\delta)} e^{u_{\alpha}} \mathrm{dv}_{g}}{\int_{M} e^{u_{\alpha}} \mathrm{dv}_{g}}=k<1 .
$$

We then get, by (15),

$$
\int_{B_{x_{0}}(\delta / 2)} e^{u_{\alpha}} \mathrm{dv}_{g} \leq C
$$

hence, since $\lim _{\alpha \rightarrow+\infty} \int_{M} e^{u_{\alpha}} \operatorname{dv}_{g}=+\infty$ by $(9)$,

$$
\varlimsup_{\alpha \rightarrow+\infty} \frac{\int_{B_{x_{0}}(\delta / 2)} e^{u_{\alpha}} \mathrm{dv}_{g}}{\int_{M} e^{u_{\alpha}} \mathrm{dv}_{g}}=0,
$$


which contradicts the fact that $x_{0}$ is a concentration point. As a consequence, we can extract a subsequence of $\left(u_{\alpha}\right)$ still denoted $\left(u_{\alpha}\right)$ such that

$$
\lim _{\alpha \rightarrow+\infty} \frac{\int_{B_{x_{0}}(\delta)} e^{u_{\alpha}} \mathrm{dv}_{g}}{\int_{M} e^{u_{\alpha}}}=1,
$$

and for that subsequence, there is one and only one concentration point.

A direct consequence is that

$$
\lim _{\alpha \rightarrow+\infty} \frac{\int_{M \backslash B_{x_{0}}(\delta)} e^{u_{\alpha}} \mathrm{dv}_{g}}{\int_{M} e^{u_{\alpha}} \mathrm{dv}_{g}}=0 .
$$

In what follows, we consider a subsequence $\left(u_{\alpha}\right)$ for which there is only one concentration point.

Step 3. We claim that, for any compact $K \subset M \backslash\left\{x_{0}\right\}$

$$
\lim _{\alpha \rightarrow+\infty} \frac{e^{u_{\alpha}}}{\int_{M} e^{u_{\alpha}} \mathrm{dv}_{g}}=0
$$

in $L^{\infty}(K)$.

Indeed, by (15) and (22) we have for any $p \geq 1$

$$
\int_{M \backslash B_{x_{0}}(\delta)} e^{p u_{\alpha}} \mathrm{dv}_{g} \leq C .
$$

Hence,

$$
\lim _{\alpha \rightarrow+\infty} \frac{\int_{M \backslash B_{x_{0}}(\delta)} e^{p u_{\alpha}} \mathrm{dv}_{g}}{\int_{M} e^{u_{\alpha}} \mathrm{dv}_{g}}=0
$$

and

$$
\lim _{\alpha \rightarrow+\infty} \frac{e^{u_{\alpha}}}{\int_{M} e^{u_{\alpha}} \mathrm{dv}_{g}}=0
$$

in $L^{p}(K)$.

From (7) we deduce that $u_{\alpha}$ is a weak solution of

$$
n \mu_{n} \Delta_{n} u_{\alpha}+\tilde{\varepsilon} n u_{\alpha}^{n-1}=\lambda_{\alpha} \frac{e^{u_{\alpha}}}{\int_{M} e^{u_{\alpha}} \mathrm{dv}_{g}} .
$$

Writing

we get the equation

$$
v_{\alpha}=\frac{e^{u_{\alpha}}}{\int_{M} e^{u_{\alpha}} \mathrm{dv}_{g}}
$$

$$
n \mu_{n} \Delta_{n} v_{\alpha}+n \mu_{n}(n-1) \frac{\left|\nabla v_{\alpha}\right|^{n}}{v_{\alpha}}+\tilde{\varepsilon} n v_{\alpha}^{n-1} u_{\alpha}^{n-1}=\lambda_{\alpha} v_{\alpha}^{n} .
$$

Since $v_{\alpha}>0, v_{\alpha} \rightarrow 0$ in $L^{p}(K)$ as $\alpha \rightarrow \infty$, and $u_{\alpha} \geq 0$, we then have weakly

$$
n \mu_{n} \Delta_{n} v_{\alpha} \leq \lambda_{\alpha} v_{\alpha}^{n} .
$$

The conclusion then follows directly from an argument of Serrin [20] (see also Trudinger [22] or Véron [23]).

Step 4. We now state the fundamental inequality of our proof. There exists $C$ such that for any $\alpha$, for any $x \in M$

$$
e^{u_{\alpha}(x)} \leq C \frac{\int_{M} e^{u_{\alpha}} \mathrm{dv}_{g}}{\mathrm{~d}_{g}^{n}\left(x, x_{\alpha}\right)}
$$


where $x_{\alpha}$ is such that $u_{\alpha}\left(x_{\alpha}\right)=\left\|u_{\alpha}\right\|_{\infty}$, and $\mathrm{d}_{g}(\cdot, \cdot)$ is the Riemannian distance. We note that according to Step $3, x_{\alpha}$ goes to $x_{0}$ as $\alpha \rightarrow+\infty$. The proof of this inequality will take several steps. In this section we "set the scene" for the proof.

We proceed by contradiction. Let

$$
z_{\alpha}(x)=\mathrm{d}_{g}^{n}\left(x, x_{\alpha}\right) \frac{e^{u_{\alpha}(x)}}{\int_{M} e^{u_{\alpha}} \mathrm{dv}_{g}} .
$$

If (23) is false, then there exists $y_{\alpha}$ such that $z_{\alpha}\left(y_{\alpha}\right)=\left\|z_{\alpha}\right\|_{\infty}$ and

$$
\lim _{\alpha \rightarrow+\infty} z_{\alpha}\left(y_{\alpha}\right)=+\infty \text {. }
$$

We do a blow up argument of the coefficient $k_{\alpha}=\mathrm{d}_{g}^{-1}\left(x_{\alpha}, y_{\alpha}\right)$. Precisely, we consider $B_{y_{\alpha}}(\delta)$ a ball of radius $\delta$ centered in $y_{\alpha}, \delta$ and $\alpha$ such that both $x_{\alpha}$ and $y_{\alpha}$ are in $B_{y_{\alpha}}(\delta)$, and the maps

$$
\begin{aligned}
\exp _{y_{\alpha}}^{-1}: \quad B_{y_{\alpha}}(\delta) \subset M & \rightarrow B_{0}(\delta) \subset \mathbb{R}^{n}, \\
x & \mapsto \bar{x} \\
y_{\alpha} & \mapsto \bar{y}_{\alpha}=0
\end{aligned}
$$

and

$$
\begin{aligned}
\Psi_{k_{\alpha}}: \quad B_{0}(\delta) & \rightarrow B_{\alpha} \subset \mathbb{R}^{n}, \\
\bar{x} & \mapsto k_{\alpha} \bar{x} .
\end{aligned}
$$

By Step 3, (24) and since $x_{\alpha} \rightarrow x_{0}$, we get that $\mathrm{d}_{g}\left(x_{\alpha}, y_{\alpha}\right) \rightarrow 0$ when $\alpha \rightarrow+\infty$, which gives that $k_{\alpha} \rightarrow+\infty$.

We also consider the metrics $g_{\alpha}=\exp _{y_{\alpha}}^{*} g\left(\right.$ on $\left.B_{0}(\delta)\right)$ and $\bar{g}_{\alpha}=k_{\alpha}^{2}\left(\Psi_{k_{\alpha}}^{-1}\right)^{*} g_{\alpha}$ (on $B_{\alpha}$ ). We get $\mathrm{d}_{\bar{g}_{\alpha}}\left(\bar{x}_{\alpha}, \bar{y}_{\alpha}\right)=1$. Finally, we consider the functions $\bar{u}_{\alpha}=u_{\alpha}$ 。 $\exp _{y_{\alpha}} \circ \Psi_{k_{\alpha}}^{-1}$ and

$$
\begin{aligned}
t_{\alpha} & =\bar{u}_{\alpha}-u_{\alpha}\left(y_{\alpha}\right)+\ln \left(\frac{\mathrm{d}_{g}^{n}\left(x_{\alpha}, y_{\alpha}\right) e^{u_{\alpha}\left(y_{\alpha}\right)}}{\int_{M} e^{u_{\alpha}} \mathrm{dv}_{g}}\right) \\
& =\bar{u}_{\alpha}+\ln \left(\frac{k_{\alpha}^{-n}}{\int_{M} e^{u_{\alpha}} \mathrm{dv}_{g}}\right) .
\end{aligned}
$$

According to (24),

$$
\lim _{\alpha \rightarrow+\infty} t_{\alpha}\left(\bar{y}_{\alpha}\right)=\ln \left(\frac{\mathrm{d}_{g}^{n}\left(x_{\alpha}, y_{\alpha}\right) e^{u_{\alpha}\left(y_{\alpha}\right)}}{\int_{M} e^{u_{\alpha}} \mathrm{dv}_{g}}\right)=+\infty .
$$

We have

$$
\Delta_{n, \bar{g}_{\alpha}} t_{\alpha}=k_{\alpha}^{-n} \Delta_{n} u_{\alpha} \text { and } \operatorname{dv}_{\bar{g}_{\alpha}}=k_{\alpha}^{n} \mathrm{dv}_{g} .
$$

Going back to $\left(E_{\alpha}\right)$ we get that $t_{\alpha}$ is a weak solution of

$$
n \mu_{n} \Delta_{n, \bar{g}_{\alpha}} t_{\alpha}+n \tilde{\varepsilon} k_{\alpha}^{-n} \bar{u}_{\alpha}^{n-1}=e^{t_{\alpha}}=\frac{e^{\bar{u}_{\alpha}}}{k_{\alpha}^{n} \int_{M} e^{u_{\alpha}} \mathrm{dv}_{g}} .
$$

We set $f_{\alpha}=e^{t_{\alpha}}$ and we get

$$
\lim _{\alpha \rightarrow+\infty} f_{\alpha}\left(\bar{y}_{\alpha}\right)=+\infty
$$

and

$$
\left(E_{\alpha}^{\prime}\right) \quad n \mu_{n} \Delta_{n, \bar{g}_{\alpha}} f_{\alpha}+(n-1) n \mu_{n} \frac{\left|\nabla f_{\alpha}\right| \overline{\bar{g}}_{\alpha}}{f_{\alpha}}+n \tilde{\varepsilon} k_{\alpha}^{-n} f_{\alpha}^{n-1} \bar{u}_{\alpha}^{n-1}=\lambda_{\alpha} f_{\alpha}^{n} .
$$


Step 5. We claim that

$$
\int_{B_{\bar{y}_{\alpha}}(R)} f_{\alpha} \mathrm{dv}_{\bar{g}_{\alpha}} \leq k<1
$$

for a set $R<1$. For that purpose, we show that there exists $r<1$ and $\gamma>0$ such that

$$
\int_{B_{\bar{x}_{\alpha}}(r)} f_{\alpha} \mathrm{dv}_{\bar{g}_{\alpha}}>\gamma
$$

This implies (26). Indeed

$$
1=\int_{B_{\alpha}} f_{\alpha} \mathrm{dv}_{\bar{g}_{\alpha}}=\int_{B_{\bar{x}_{\alpha}}(r)} f_{\alpha} \mathrm{dv}_{\bar{g}_{\alpha}}+\int_{B_{\alpha} \backslash B_{\bar{x}_{\alpha}}(r)} f_{\alpha} \mathrm{dv}_{\bar{g}_{\alpha}}
$$

and we can set $r$ and $R$ such that $B_{\bar{x}_{\alpha}}(r) \cap B_{\bar{y}_{\alpha}}(R)=\emptyset$ since $\mathrm{d}_{\bar{g}_{\alpha}}\left(\bar{x}_{\alpha}, \bar{y}_{\alpha}\right)=1$.

We do a blow up argument of the coefficient

$$
k_{\alpha}^{\prime n}=\frac{e^{u_{\alpha}\left(x_{\alpha}\right)}}{\int_{M} e^{u_{\alpha}} \mathrm{dv}_{g}} .
$$

We keep the same maps $\left(\exp _{y_{\alpha}}^{-1}, \Psi_{k_{\alpha}}\right)$ and notations $\left(B_{y_{\alpha}}, B_{\alpha}, \bar{x}_{\alpha}, \bar{y}_{\alpha}, \ldots\right)$ as in Step 4. We have

$$
\lim _{\alpha \rightarrow+\infty} \frac{e^{u_{\alpha}\left(y_{\alpha}\right)}}{\int_{M} e^{u_{\alpha}} \mathrm{dv}_{g}}=+\infty
$$

by (24) and since $\mathrm{d}_{g}^{n}\left(x_{\alpha}, y_{\alpha}\right) \rightarrow 0$ when $\alpha \rightarrow+\infty$. Since

$$
\frac{e^{u_{\alpha}\left(x_{\alpha}\right)}}{\int_{M} e^{u_{\alpha}} \mathrm{dv} g_{g}} \geq \frac{e^{u_{\alpha}\left(y_{\alpha}\right)}}{\int_{M} e^{u_{\alpha}} \mathrm{dv}_{g}}
$$

we get that $k_{\alpha}^{\prime} \rightarrow+\infty$ when $\alpha \rightarrow+\infty$.

For this new blow up, we consider the metrics

$$
g_{\alpha}{ }^{\prime}=\exp _{x_{\alpha}}^{*} g \text { and } \bar{g}_{\alpha}^{\prime}=k_{\alpha}^{\prime 2}\left(\Psi_{k_{\alpha}^{\prime}}^{-1}\right)^{*} g_{\alpha}{ }^{\prime},
$$

and the functions $\bar{v}_{\alpha}=\bar{u}_{\alpha}-u_{\alpha}\left(x_{\alpha}\right)\left(\bar{u}_{\alpha}\right.$ as in point 4). We note that $\bar{v}_{\alpha}\left(\bar{x}_{\alpha}\right)=0$.

Then $\bar{v}_{\alpha}$ is a weak solution of

$$
n \mu_{n} \Delta_{n, \bar{g}_{\alpha}^{\prime}} \bar{v}_{\alpha}+n \tilde{\varepsilon} k_{\alpha}^{\prime-n} \bar{u}_{\alpha}^{n-1}=\lambda_{\alpha} e^{\bar{v}_{\alpha}} .
$$

We set $w_{\alpha}=e^{\bar{v}_{\alpha}}$ and we get $w_{\alpha}\left(\bar{x}_{\alpha}\right)=1,0<w_{\alpha} \leq 1$ and

$$
\left(E_{\alpha}^{\prime \prime}\right) \quad n \mu_{n} \Delta_{n, \bar{g}_{\alpha}^{\prime}} w_{\alpha}+(n-1) n \mu_{n} \frac{\left|\nabla w_{\alpha}\right|_{\bar{g}_{\alpha}^{\prime}}}{w_{\alpha}}+n \tilde{\varepsilon} k_{\alpha}^{\prime-n} w_{\alpha}^{n-1} \bar{u}_{\alpha}^{n-1}=\lambda_{\alpha} w_{\alpha}^{n} .
$$

On one hand, we have

$$
\int_{B_{\bar{x}_{\alpha}}(r)} w_{\alpha} \mathrm{dv}_{\bar{g}_{\alpha}^{\prime}}=\int_{B_{x_{\alpha}}\left(r k_{\alpha}^{\prime-1}\right)} \frac{e^{u_{\alpha}}}{e^{u_{\alpha}\left(x_{\alpha}\right)}} \frac{e^{u_{\alpha}\left(x_{\alpha}\right)}}{\int_{M} e^{u_{\alpha}} \mathrm{dv}_{g}} \mathrm{dv}_{g}=\frac{\int_{B_{x_{\alpha}}\left(r k_{\alpha}^{\prime-1}\right)} e^{u_{\alpha}} \mathrm{dv}_{g}}{\int_{M} e^{u_{\alpha}} \mathrm{dv}_{g}} .
$$

On the other hand, we get, the same way,

$$
\int_{B_{\bar{x}_{\alpha}}(r)} f_{\alpha} \mathrm{dv}_{\bar{g}_{\alpha}}=\frac{\int_{B_{x_{\alpha}}\left(r k_{\alpha}^{-1}\right)} e^{u_{\alpha}} \mathrm{dv}_{g}}{\int_{M} e^{u_{\alpha}} \mathrm{dv}_{g}}
$$


with $f_{\alpha}$ and $k_{\alpha}$ as in Step 4. But

$$
k_{\alpha}^{\prime n}=\frac{e^{u_{\alpha}\left(x_{\alpha}\right)}}{\int_{M} e^{u_{\alpha}} \mathrm{dv}_{g}}>k_{\alpha}^{n}=\frac{1}{\mathrm{~d}^{n}\left(x_{\alpha}, y_{\alpha}\right)} .
$$

Indeed: $k_{\alpha}^{\prime n}>k_{\alpha}^{n}$ means

$$
\mathrm{d}_{g}^{n}\left(x_{\alpha}, y_{\alpha}\right) \frac{e^{u_{\alpha}\left(x_{\alpha}\right)}}{\int_{M} e^{u_{\alpha}} \mathrm{dv}_{g}}>1
$$

and

$$
\mathrm{d}_{g}^{n}\left(x_{\alpha}, y_{\alpha}\right) \frac{e^{u_{\alpha}\left(x_{\alpha}\right)}}{\int_{M} e^{u_{\alpha}} \mathrm{dv}_{g}}>\mathrm{d}_{g}^{n}\left(x_{\alpha}, y_{\alpha}\right) \frac{e^{u_{\alpha}\left(y_{\alpha}\right)}}{\int_{M} e^{u_{\alpha}} \mathrm{dv}_{g}}>1
$$

is true for $\alpha$ big enough according to (24). Hence, $k_{\alpha}^{\prime}>k_{\alpha}$, which gives $B_{x_{\alpha}}\left(r k_{\alpha}^{\prime-1}\right)$ $\subset B_{x_{\alpha}}\left(r k_{\alpha}^{-1}\right)$, and in order to prove (27), with (28) and (29) one just has to prove that there exists $\gamma>0$ such that $\int_{B_{\bar{x}_{\alpha}}(r)} w_{\alpha} \mathrm{dv}_{\bar{g}_{\alpha}^{\prime}}>\gamma$.

We first prove that $\int_{B_{\bar{x}_{\alpha}}(r)} w_{\alpha}^{n} \mathrm{dv}_{\bar{g}_{\alpha}^{\prime}}>\gamma$. We proceed by contradiction. Assume that $\lim _{\alpha \rightarrow+\infty} \int_{B_{\bar{x}_{\alpha}}(r)} w_{\alpha}^{n} \operatorname{dv}_{\bar{g}_{\alpha}^{\prime}}=0$. We know that $w_{\alpha}$ verifies $\left(E_{\alpha}^{\prime \prime}\right)$, which precisely means, for any $\Phi \in H_{1}^{n}\left(B_{\alpha}\right)$

$$
n \mu_{n} \int_{B_{\alpha}} \frac{\left|\nabla w_{\alpha}\right|^{n-2} \nabla w_{\alpha} \nabla \Phi}{w_{\alpha}^{n-1}} \operatorname{dv}_{\bar{g}_{\alpha}^{\prime}}+\tilde{\varepsilon} n k_{\alpha}^{\prime-n} \int_{B_{\alpha}} \bar{u}_{\alpha}^{n-1} \Phi \operatorname{dv}_{\bar{g}_{\alpha}^{\prime}}=\lambda_{\alpha} \int_{B_{\alpha}} w_{\alpha} \Phi \operatorname{dv}_{\bar{g}_{\alpha}^{\prime}} .
$$

We test $\Phi=\eta^{n} w_{\alpha}^{n}$, where $\eta$ is a $\mathcal{C}^{\infty}\left(B_{\alpha}\right)$ cut off function of $B_{\bar{x}_{\alpha}}(r / 2), \eta=1$ on $B_{\bar{x}_{\alpha}}(r / 2)$ and $\eta=0$ on $B_{\alpha} \backslash B_{\bar{x}_{\alpha}}(r)$ (since $B_{\bar{x}_{\alpha}}(r / 2)$ depends on $\alpha$, our cut off function should depend on $\alpha$, but it does not affect the boundaries we make, so we still denote it $\eta$ ). We get

$$
\begin{array}{r}
C \int_{B_{\alpha}}\left|\nabla w_{\alpha}\right| \bar{g}_{\alpha}^{\prime} \eta^{n} \operatorname{dv}_{\bar{g}_{\alpha}^{\prime}}+C \int_{B_{\alpha}} w_{\alpha}\left|\nabla w_{\alpha}\right|^{n-2} \nabla w_{\alpha} \nabla\left(\eta^{n}\right) \mathrm{dv}_{\bar{g}_{\alpha}^{\prime}} \\
+C k_{\alpha}^{\prime-n} \int_{B_{\alpha}} \eta^{n} w_{\alpha}^{n} \bar{u}_{\alpha}^{n-1} \operatorname{dv}_{\bar{g}_{\alpha}}=\lambda_{\alpha} \int_{B_{\alpha}} \eta w_{\alpha}^{n+1} .
\end{array}
$$

We have

$$
\left.C_{1} \int_{B_{\alpha}}\left|\nabla w_{\alpha}\right|\right|_{\bar{g}_{\alpha}^{\prime}} ^{n} \eta^{n} \operatorname{dv}_{\bar{g}_{\alpha}^{\prime}} \geq C_{2}\left(\int_{B_{\alpha}}\left|\nabla\left(\eta w_{\alpha}\right)\right|^{n} \operatorname{dv}_{\bar{g}_{\alpha}^{\prime}}-\int_{B_{\alpha}} w_{\alpha}^{n}|\nabla \eta|^{n} \mathrm{dv}_{\bar{g}_{\alpha}^{\prime}}\right)
$$

and

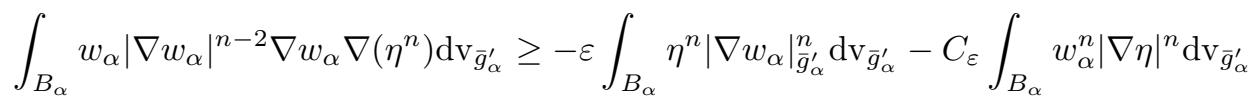

so with a set $\varepsilon$,

$$
\begin{array}{r}
C \int_{B_{\alpha}}\left|\nabla w_{\alpha}\right|_{\bar{g}_{\alpha}^{\prime}}^{n} \eta^{n} \operatorname{dv}_{\bar{g}_{\alpha}^{\prime}}+C \int_{B_{\alpha}} w_{\alpha}\left|\nabla w_{\alpha}\right|^{n-2} \nabla w_{\alpha} \nabla\left(\eta^{n}\right) \mid \operatorname{dv}_{\bar{g}_{\alpha}^{\prime}} \\
\geq C \int_{B_{\alpha}}\left|\nabla\left(\eta w_{\alpha}\right)\right|^{n} \operatorname{dv}_{\bar{g}_{\alpha}^{\prime}}-C \int_{B_{\alpha}} w_{\alpha}^{n}|\nabla \eta|^{n} \operatorname{dv}_{\bar{g}_{\alpha}^{\prime}} .
\end{array}
$$

On the other hand, since $w_{\alpha} \leq 1$

$$
\int_{B_{\alpha}} \eta^{n} w_{\alpha}^{n+1} \mathrm{dv}_{\bar{g}_{\alpha}^{\prime}} \leq \int_{B_{\alpha}} \eta^{n} w_{\alpha}^{n} \mathrm{dv}_{\bar{g}_{\alpha}^{\prime}}
$$

Hence

$$
C \int_{B_{\alpha}}\left|\nabla\left(\eta w_{\alpha}\right)\right|^{n} \mathrm{dv}_{\bar{g}_{\alpha}^{\prime}} \leq C \int_{B_{\alpha}} \eta^{n} w_{\alpha}^{n} \operatorname{dv}_{\bar{g}_{\alpha}^{\prime}}+C \int_{B_{\alpha}} w_{\alpha}^{n}|\nabla \eta|^{n} \mathrm{dv}_{\bar{g}_{\alpha}^{\prime}},
$$


which leads to

$$
C \int_{B_{\alpha}}\left|\nabla\left(\eta w_{\alpha}\right)\right|^{n} \mathrm{dv}_{\bar{g}_{\alpha}^{\prime}} \leq C \int_{B_{\bar{x}_{\alpha}}(r)} w_{\alpha}^{n} \mathrm{dv}_{\bar{g}_{\alpha}^{\prime}}
$$

and $\lim _{\alpha \rightarrow+\infty} \int_{B_{\bar{x}_{\alpha}}(r)} w_{\alpha}^{n} \operatorname{dv}_{\bar{g}_{\alpha}^{\prime}}=0$ by our initial assumption, which gives

$$
\lim _{\alpha \rightarrow+\infty} \int_{B_{\bar{x}_{\alpha}}(r)}\left|\nabla\left(\eta w_{\alpha}\right)\right|^{n} \operatorname{dv}_{\bar{g}_{\alpha}^{\prime}}=0 .
$$

Since for any $p \geq 1$, with the compact embeding $H_{1}^{n} \subset L^{p}$

$$
\left(\int_{B_{\alpha}}\left(\eta w_{\alpha}\right)^{p} \mathrm{dv}_{\bar{g}_{\alpha}^{\prime}}\right)^{n / p} \leq \varepsilon \int_{B_{\alpha}}\left|\nabla\left(\eta w_{\alpha}\right)\right|^{n} \operatorname{dv}_{\bar{g}_{\alpha}^{\prime}}+C_{\varepsilon} \int_{B_{\alpha}}\left(\eta w_{\alpha}\right)^{n} \operatorname{dv}_{\bar{g}_{\alpha}^{\prime}}
$$

we get that $w_{\alpha} \rightarrow 0$ in $L^{p}\left(B_{\bar{x}_{\alpha}}(r / 2)\right)$ for any $p \geq 1$. As shown by Serrin [20], we get that $w_{\alpha} \rightarrow 0$ in $L^{\infty}\left(B_{\bar{x}_{\alpha}}(r / 2)\right)$, which is absurd since $w_{\alpha}\left(\bar{x}_{\alpha}\right)=1$. Hence our initial assumption must be false, and

$$
\int_{B_{\bar{x}_{\alpha}}(r)} w_{\alpha}^{n} \mathrm{dv}_{\bar{g}_{\alpha}^{\prime}}>\gamma .
$$

We now claim that $\int_{B_{\bar{x}_{\alpha}}(r)} w_{\alpha} \operatorname{dv}_{\bar{g}_{\alpha}^{\prime}}>\gamma$. With the same $\eta$, we still have

$$
\begin{gathered}
C \int_{B_{\alpha}} \frac{\left|\nabla w_{\alpha}\right|^{n-2} \nabla w_{\alpha} \nabla\left(\eta^{n} w_{\alpha}^{n}\right)}{w_{\alpha}^{n-1}} \mathrm{dv}_{\bar{g}_{\alpha}^{\prime}}+C \int_{B_{\alpha}} \bar{u}_{\alpha}^{n-1} \eta^{n} w_{\alpha}^{n} \operatorname{dv}_{\bar{g}_{\alpha}^{\prime}} \\
\quad=\lambda_{\alpha} \int_{B_{\alpha}} \eta^{n} w_{\alpha}^{n+1} \operatorname{dv}_{\bar{g}_{\alpha}^{\prime}} \leq C
\end{gathered}
$$

because $0<w_{\alpha} \leq 1$ and $\eta$ has compact support. Since $\bar{u}_{\alpha}^{n-1} w_{\alpha}^{n} \geq 0$ we get

$$
C \int_{B_{\alpha}}\left|\nabla w_{\alpha}\right| \frac{n}{\bar{g}_{\alpha}^{\prime}} \eta^{n} \mathrm{dv}_{\bar{g}_{\alpha}^{\prime}}+C \int_{B_{\alpha}} w_{\alpha} \eta^{n-1}\left|\nabla w_{\alpha}\right|^{n-2} \nabla w_{\alpha} \nabla \eta \mathrm{dv}_{\bar{g}_{\alpha}^{\prime}} \leq C .
$$

For any $\varepsilon>0$ there exists $C_{\varepsilon}>0$ such that

$\int_{B_{\alpha}} \eta^{n-1} w_{\alpha}\left|\nabla w_{\alpha}\right|^{n-2} \nabla w_{\alpha} \nabla \eta \mathrm{dv}_{\bar{g}_{\alpha}^{\prime}} \geq-\left.\varepsilon \int_{B_{\alpha}} \eta^{n}\left|\nabla w_{\alpha}\right|\right|_{\bar{g}_{\alpha}^{\prime}} ^{n} \mathrm{dv}_{\bar{g}_{\alpha}^{\prime}}-C_{\varepsilon} \int_{B_{\alpha}} w_{\alpha}^{n}|\nabla \eta|^{n} \mathrm{dv}_{\bar{g}_{\alpha}^{\prime}}$ and since $w_{\alpha} \leq 1$, and by definition of $\eta$,

$$
\int_{B_{\alpha}} w_{\alpha}^{n}|\nabla \eta|^{n} \mathrm{dv}_{\bar{g}_{\alpha}^{\prime}} \leq C \int_{B_{x_{\alpha}}(r)} 1 \mathrm{dv}_{\bar{g}_{\alpha}^{\prime}} \leq C .
$$

Hence for a small $\varepsilon$ we get

$$
\int_{B_{\alpha}}\left|\nabla w_{\alpha}\right|_{\bar{g}_{\alpha}^{\prime}}^{n} \eta^{n} \operatorname{dv}_{\bar{g}_{\alpha}^{\prime}} \leq C
$$

and

$$
\int_{B_{\alpha}}\left|\nabla\left(\eta w_{\alpha}\right)\right|^{n} \mathrm{dv}_{\bar{g}_{\alpha}^{\prime}} \leq C .
$$

Since the embedding $H_{1}^{n} \subset L^{n}$ is compact

$$
\int_{B_{\alpha}} \eta^{n} w_{\alpha}^{n} \mathrm{dv}_{\bar{g}_{\alpha}^{\prime}} \leq \varepsilon \int_{B_{\alpha}}\left|\nabla\left(\eta w_{\alpha}\right)\right|^{n} \mathrm{dv}_{\bar{g}_{\alpha}^{\prime}}+C_{\varepsilon}\left(\int_{B_{\alpha}} \eta w_{\alpha} \mathrm{dv}_{\bar{g}_{\alpha}^{\prime}}\right)^{n} .
$$

We've already proved that $\int_{B_{\alpha}} \eta^{n} w_{\alpha}^{n} \operatorname{dv}_{\bar{g}_{\alpha}^{\prime}}>\gamma>0$ and $\int_{B_{\alpha}}\left|\nabla\left(\eta w_{\alpha}\right)\right|^{n} \operatorname{dv}_{\bar{g}_{\alpha}^{\prime}} \leq C$, hence there exists $\gamma^{\prime}>0$ such that

$$
\int_{B_{\bar{x}_{\alpha}}(r)} \eta w_{\alpha} \operatorname{dv}_{\bar{g}_{\alpha}^{\prime}}>\gamma^{\prime}
$$


Going back to what we said at the beginning of Step 5, we have proved that

$$
\int_{B_{\bar{y}_{\alpha}}(R)} f_{\alpha} \mathrm{dv}_{\bar{g}_{\alpha}} \leq k<1 .
$$

Step 6. We now prove that there exists $\delta>0$

$$
\int_{B_{\bar{y}_{\alpha}}(R)} f_{\alpha}^{1+\delta} \mathrm{dv}_{\bar{g}_{\alpha}} \leq C .
$$

With the same notations as in Step 4

$$
\begin{aligned}
\int_{B_{\bar{y}_{\alpha}}(R)} f_{\alpha}^{1+\delta} \operatorname{dv}_{\bar{g}_{\alpha}} & =\int_{B_{y_{\alpha}}\left(R k_{\alpha}^{-1}\right)}\left(\frac{e^{u_{\alpha}}}{k_{\alpha}^{n} \int_{M} e^{u_{\alpha}} \mathrm{dv}_{g}}\right)^{1+\delta} k_{\alpha}^{n} \mathrm{dv}_{g} \\
& =\frac{1}{k_{\alpha}^{n \delta}\left(\int_{M} e^{u_{\alpha}} \mathrm{dv}_{g}\right)^{\delta}} \frac{\int_{B_{y_{\alpha}}\left(R k_{\alpha}^{-1}\right)} e^{u_{\alpha}(1+\delta)} \mathrm{dv}_{g}}{\int_{M} e^{u_{\alpha}} \mathrm{dv}_{g}}
\end{aligned}
$$

By Step 5 we know

$$
\begin{aligned}
\int_{B_{\bar{y}_{\alpha}}(R)} f_{\alpha} \operatorname{dv}_{\bar{g}_{\alpha}} & =\int_{B_{y_{\alpha}}\left(R k_{\alpha}^{-1}\right)} \frac{e^{u_{\alpha}}}{k_{\alpha}^{n} \int_{M} e^{u_{\alpha}} \mathrm{dv}_{g}} k_{\alpha}^{n} \mathrm{dv}_{g} \\
& =\frac{\int_{B_{y_{\alpha}}\left(R k_{\alpha}^{-1}\right)} e^{u_{\alpha}} \mathrm{dv}_{g}}{\int_{M} e^{u_{\alpha}} \mathrm{dv}_{g}} \leq k<1
\end{aligned}
$$

with (26).

In (8) we test $\Phi^{2}=\eta_{\alpha}^{n} e^{\delta u_{\alpha}}$ where $\eta_{\alpha}$ is a cut off function of $B_{y_{\alpha}}\left(R k_{\alpha}^{-1} / 2\right), \eta_{\alpha}=1$ on $B_{y_{\alpha}}\left(R k_{\alpha}^{-1} / 2\right), \eta_{\alpha}=0$ on $M \backslash B_{y_{\alpha}}\left(R k_{\alpha}^{-1}\right), 0 \leq \eta_{\alpha} \leq 1$ and $\left|\nabla \eta_{\alpha}\right| \leq C k_{\alpha}$.

We note that $B_{y_{\alpha}}\left(R k_{\alpha}^{-1}\right)$ is a ball with a radius going to 0 with $\alpha$, since $k_{\alpha} \rightarrow+\infty$ when $\alpha \rightarrow+\infty$.

Together with (26), and with the same calculations as in the proof of (14) we find

$$
\begin{array}{r}
\lambda_{\alpha}(1-k) \frac{\int_{M} \eta_{\alpha}^{n} e^{u_{\alpha}(1+\delta)} \mathrm{dv}_{g}}{\int_{M} e^{u_{\alpha}} \mathrm{dv}_{g}} \leq C \int_{M}\left|\nabla u_{\alpha}\right|^{n-2}\left|\nabla\left(\eta_{\alpha}^{\frac{n}{2}} e^{\frac{\delta u_{\alpha}}{2}}\right)\right|^{2} \mathrm{dv}_{g} \\
+C \int_{M} u_{\alpha}^{n-2} \eta_{\alpha}^{n} e^{\delta u_{\alpha}} \mathrm{dv}_{g}
\end{array}
$$

In (7) we test $\Phi=\eta_{\alpha}^{n} e^{\delta u_{\alpha}}$ which gives

$C \int_{M}\left|\nabla u_{\alpha}\right|^{n-2} \nabla u_{\alpha} \nabla\left(\eta_{\alpha}^{n} e^{\delta u_{\alpha}}\right) \mathrm{dv}_{g}+C \int_{M} u_{\alpha}^{n-1} \eta_{\alpha}^{n} e^{\delta u_{\alpha}} \operatorname{dv}_{g}=\lambda_{\alpha} \frac{\int_{M} \eta_{\alpha}^{n} e^{u_{\alpha}(1+\delta)} \mathrm{dv}_{g}}{\int_{M} e^{u_{\alpha}} \mathrm{dv}_{g}}$.

We set $A=\int_{M}\left|\nabla u_{\alpha}\right|^{n-2}\left|\nabla\left(\eta_{\alpha}^{\frac{n}{2}} e^{\frac{\delta u_{\alpha}}{2}}\right)\right|^{2} \operatorname{dv}_{g}$. We get

$$
\begin{aligned}
A= & \int_{M}\left|\nabla u_{\alpha}\right|^{n-2}\left|\nabla \eta_{\alpha}^{\frac{n}{2}}\right|^{2} e^{\delta u_{\alpha}} \mathrm{dv}_{g}+\frac{\delta^{2}}{4} \int_{M}\left|\nabla u_{\alpha}\right|^{n} \eta_{\alpha}^{n} e^{\delta u_{\alpha}} \mathrm{dv}_{g} \\
& +\delta \int_{M}\left|\nabla u_{\alpha}\right|^{n-2} e^{\delta u_{\alpha}} \eta_{\alpha}^{\frac{n}{2}} \nabla u_{\alpha} \nabla \eta_{\alpha}^{\frac{n}{2}} \mathrm{dv}_{g}
\end{aligned}
$$

and

$$
\delta \int_{M}\left|\nabla u_{\alpha}\right|^{n-2} \nabla u_{\alpha} \nabla\left(\eta_{\alpha}^{n} e^{\delta u_{\alpha}}\right) \mathrm{dv}_{g}=4 A+Y
$$


where

$$
\begin{array}{r}
Y=\delta \int_{M}\left|\nabla u_{\alpha}\right|^{n-2} \nabla u_{\alpha} \nabla \eta_{\alpha}^{n} e^{\delta u_{\alpha}} \operatorname{dv}_{g}-C \underbrace{\int_{M}\left|\nabla u_{\alpha}\right|^{n-2}\left|\nabla \eta_{\alpha}^{\frac{n}{2}}\right|^{2} e^{\delta u_{\alpha}} \mathrm{dv}_{g}}_{I_{1}} \\
-C \delta \underbrace{\int_{M}\left|\nabla u_{\alpha}\right|^{n-2} \eta_{\alpha}^{\frac{n}{2}} e^{\delta u_{\alpha}} \nabla u_{\alpha} \nabla \eta_{\alpha}^{\frac{n}{2}} \mathrm{dv} g}_{I_{2}}
\end{array}
$$

and all constants $C$ are positive and independent of $\alpha$ and $\delta$. We compute (34) in (33), and since $\int_{M} u_{\alpha}^{n-1} \eta_{\alpha}^{n} e^{\delta u_{\alpha}} \mathrm{dv}_{g} \geq 0, \lambda_{\alpha}<1$ we get

$$
C Y+C A \leq \delta \frac{\int_{M} e^{(1+\delta) u_{\alpha}} \eta_{\alpha}^{n} \mathrm{dv}_{g}}{\int_{M} e^{u_{\alpha}} \operatorname{dv}_{g}} .
$$

On the other hand, we note that

$\left|\nabla u_{\alpha}\right|^{n-2} e^{\delta u_{\alpha}} \eta_{\alpha}^{\frac{n}{2}} \nabla u_{\alpha} \nabla \eta_{\alpha}^{\frac{n}{2}}=\left(\left|\nabla u_{\alpha}\right|^{\frac{n}{2}} \eta_{\alpha}^{\frac{n}{2}} e^{\frac{\delta u_{\alpha}}{2}}\right)\left(e^{\frac{\delta u_{\alpha}}{2}}\left|\nabla u_{\alpha}\right|^{-1}\left|\nabla u_{\alpha}\right|^{\frac{n-2}{2}} \nabla u_{\alpha} \nabla \eta_{\alpha}^{\frac{n}{2}}\right)$, hence for any $\varepsilon_{1}>0$, there exists $C_{\varepsilon_{1}}>0$ such that

$$
\begin{aligned}
& \int_{M}\left|\nabla u_{\alpha}\right|^{n-2} e^{\delta u_{\alpha}} \eta_{\alpha}^{\frac{n}{2}} \nabla u_{\alpha} \nabla \eta_{\alpha}^{\frac{n}{2}} \mathrm{dv}_{g} \\
& \quad \geq-\varepsilon_{1} \int_{M} \eta_{\alpha}^{n} e^{\delta u_{\alpha}}\left|\nabla u_{\alpha}\right|^{n} \mathrm{dv}_{g}-C_{\varepsilon_{1}} \int_{M}\left|\nabla u_{\alpha}\right|^{n-2}\left|\nabla \eta_{\alpha}^{\frac{n}{2}}\right|^{2} e^{\delta u_{\alpha}} \mathrm{dv}_{g} .
\end{aligned}
$$

This way

$$
\begin{aligned}
A \geq & \int_{M}\left|\nabla u_{\alpha}\right|^{n-2}\left|\nabla \eta_{\alpha}^{\frac{n}{2}}\right|^{2} e^{\delta u_{\alpha}} \mathrm{dv}_{g}+\frac{\delta^{2}}{4} \int_{M} \eta_{\alpha}^{n} e^{\delta u_{\alpha}}\left|\nabla u_{\alpha}\right|^{n} \operatorname{dv}_{g} \\
& -\varepsilon_{1} \int_{M} \eta_{\alpha}^{n} e^{\delta u_{\alpha}}\left|\nabla u_{\alpha}\right|^{n} \operatorname{dv}_{g}-C_{\varepsilon_{1}} \int_{M}\left|\nabla u_{\alpha}\right|^{n-2}\left|\nabla \eta_{\alpha}^{\frac{n}{2}}\right|^{2} e^{\delta u_{\alpha}} \mathrm{dv}_{g} \\
\geq & C \delta^{2} \int_{M} \eta_{\alpha}^{n} e^{\delta u_{\alpha}}\left|\nabla u_{\alpha}\right|^{n} \operatorname{dv}_{g}-C_{\varepsilon_{1}} \int_{M}\left|\nabla u_{\alpha}\right|^{n-2}\left|\nabla \eta_{\alpha}^{\frac{n}{2}}\right|^{2} e^{\delta u_{\alpha}} \mathrm{dv}_{g}
\end{aligned}
$$

Going back to (35) and writing $A=A / 2+A / 2$ we get

$$
\begin{array}{r}
\delta \frac{\int_{M} e^{(1+\delta) u_{\alpha}} \eta_{\alpha}^{n} \mathrm{dv}_{g}}{\int_{M} e^{u_{\alpha}} \mathrm{dv}_{g}} \geq \frac{C}{2} A+C \delta^{2} \int_{M} \eta_{\alpha}^{n} e^{\delta u_{\alpha}}\left|\nabla u_{\alpha}\right|^{n} \mathrm{dv}_{g} \\
-\frac{C_{\varepsilon_{1}}}{2} \int_{M}\left|\nabla u_{\alpha}\right|^{n-2}\left|\nabla \eta_{\alpha}^{\frac{n}{2}}\right|^{2} e^{\delta u_{\alpha}} \mathrm{dv}_{g}+C Y .
\end{array}
$$

We now study $I_{1}$ and $I_{2}$ from $Y$. For any $\varepsilon_{2}>0$, there exists $C_{\varepsilon_{2}}$ such that

$$
\begin{aligned}
I_{1} & =C \int_{M}\left|\nabla u_{\alpha}\right|^{n-2} e^{\delta u_{\alpha}} \eta_{\alpha}^{n-2}\left|\nabla \eta_{\alpha}\right|^{2} \mathrm{dv}_{g} \\
& \leq \varepsilon_{2} \int_{M}\left|\nabla u_{\alpha}\right|^{n} \eta_{\alpha}^{n} e^{\delta u_{\alpha}} \mathrm{dv}_{g}+C_{\varepsilon_{2}} \int_{M} e^{\delta u_{\alpha}}\left|\nabla \eta_{\alpha}\right|^{n} \mathrm{dv}_{g} .
\end{aligned}
$$

For any $\varepsilon_{3}>0$, there exists $C_{\varepsilon_{3}}>0$ such that

$$
\begin{aligned}
I_{2} & \leq C \int_{M}\left|\nabla u_{\alpha}\right|^{n-1} \eta_{\alpha}^{n-1} e^{\delta u_{\alpha}}\left|\nabla \eta_{\alpha}\right| \operatorname{dv}_{g} \\
& \leq \varepsilon_{3} \int_{M}\left|\nabla u_{\alpha}\right|^{n} \eta_{\alpha}^{n} e^{\delta u_{\alpha}} \operatorname{dv}_{g}+C_{\varepsilon_{3}} \int_{M} e^{\delta u_{\alpha}}\left|\nabla \eta_{\alpha}\right|^{n} \operatorname{dv}_{g} .
\end{aligned}
$$


Choosing $\varepsilon_{1}, \varepsilon_{2}, \varepsilon_{3}$ small enough regarding $\delta$ we get

$$
\delta \frac{\int_{M} e^{(1+\delta) u_{\alpha}} \eta_{\alpha}^{n} \mathrm{dv}_{g}}{\int_{M} e^{u_{\alpha}} \mathrm{dv}_{g}} \geq \frac{C}{2} A+\delta \int_{M} \eta_{\alpha}^{n} e^{\delta u_{\alpha}}\left|\nabla u_{\alpha}\right|^{n} \operatorname{dv}_{g}-C \int_{M} e^{\delta u_{\alpha}}\left|\nabla \eta_{\alpha}\right|^{n} \mathrm{dv}_{g}
$$

and finally

$$
A \leq C \delta \frac{\int_{M} e^{(1+\delta) u_{\alpha}} \eta_{\alpha}^{n} \mathrm{dv}_{g}}{\int_{M} e^{u_{\alpha}} \mathrm{dv}_{g}}+C \int_{M} e^{\delta u_{\alpha}}\left|\nabla \eta_{\alpha}\right|^{n} \mathrm{dv}_{g} .
$$

We compute (36) in (32) and with $\delta$ small enough we get

$$
\frac{\int_{M} \eta_{\alpha}^{n} e^{u_{\alpha}(1+\delta)} \mathrm{dv}_{g}}{\int_{M} e^{u_{\alpha}} \mathrm{dv}_{g}} \leq C \int_{M} e^{\delta u_{\alpha}}\left|\nabla \eta_{\alpha}\right|^{n} \mathrm{dv}_{g}+C \int_{M} u_{\alpha}^{n-2} \eta_{\alpha}^{n} e^{\delta u_{\alpha}} \mathrm{dv}_{g} .
$$

Together with (31)

$$
\int_{B_{\bar{y}_{\alpha}}(R)} f_{\alpha}^{1+\delta} \operatorname{dv}_{\bar{g}_{\alpha}} \leq C \frac{\int_{M} e^{\delta u_{\alpha}}\left|\nabla \eta_{\alpha}\right|^{n} \mathrm{dv}_{g}+\int_{M} u_{\alpha}^{n-2} \eta_{\alpha}^{n} e^{\delta u_{\alpha}} \mathrm{dv}_{g}}{k_{\alpha}^{n \delta}\left(\int_{M} e^{u_{\alpha}} \mathrm{dv}_{g}\right)^{\delta}} .
$$

On one hand, recalling that $\left|\nabla \eta_{\alpha}\right| \leq C k_{\alpha}$, and $\eta_{\alpha}=0$ on $M \backslash B_{y_{\alpha}}\left(R k_{\alpha}^{-1}\right)$ we have

$$
\begin{aligned}
\frac{\int_{M} e^{\delta u_{\alpha}}\left|\nabla \eta_{\alpha}\right|^{n} \mathrm{dv}_{g}}{k_{\alpha}^{n \delta}\left(\int_{M} e^{u_{\alpha}} \mathrm{dv}_{g}\right)^{\delta}} & \leq C \frac{k_{\alpha}^{n} \int_{B_{y_{\alpha}}\left(R k_{\alpha}^{-1}\right)} e^{\delta u_{\alpha}} \mathrm{dv}_{g}}{k_{\alpha}^{n \delta}\left(\int_{M} e^{u_{\alpha}} \mathrm{dv}_{g}\right)^{\delta}} \\
& \leq C \frac{k_{\alpha}^{n}}{k_{\alpha}^{n \delta}}\left(\int_{B_{y_{\alpha}}\left(R k_{\alpha}^{-1}\right)} 1 \mathrm{dv}_{g}\right)^{1-\delta}\left(\frac{\int_{B_{y_{\alpha}}\left(R k_{\alpha}^{-1}\right)} e^{\delta \frac{u_{\alpha}}{\delta}} \mathrm{dv}_{g}}{\int_{M} e^{u_{\alpha}} \mathrm{dv}_{g}}\right)^{\delta} \\
& \leq C \frac{k_{\alpha}^{n}}{k_{\alpha}^{n \delta} k_{\alpha}^{n(1-\delta)}}\left(\frac{\int_{B_{y_{\alpha}}\left(R k_{\alpha}^{-1}\right)} e^{u_{\alpha}} \mathrm{dv}_{g}}{\int_{M} e^{u_{\alpha}} \mathrm{dv}_{g}} \leq C .\right.
\end{aligned}
$$

On the other hand, since for any $q \geq 1 \int_{M} u_{\alpha}^{q} \mathrm{dv}_{g} \leq C$, we can choose $p>1$ such that

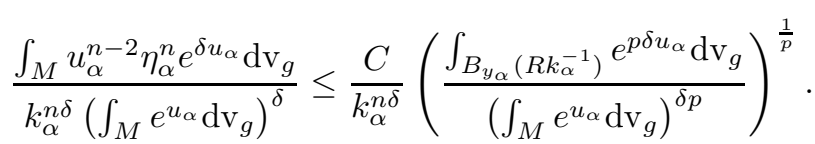

Choosing $p$ such that $p \delta=1$, with the fact that the ball has a radius that goes to 0 when $\alpha \rightarrow+\infty$, we finally get, getting back to (37)

$$
\int_{B_{\bar{y}_{\alpha}}(R)} f_{\alpha}^{(1+\delta)} \mathrm{dv}_{\bar{g}_{\alpha}} \leq C .
$$

Inequality (30) is proven.

Step 7. We are (finally!) ready to prove inequality (23). We recall that we proceeded by contradiction. We get our result by proving that $f_{\alpha}$ is bounded in $L^{\infty}\left(B_{\bar{y}_{\alpha}}(R / 2)\right.$ ), when by construction (Step 4$), f_{\alpha}\left(\bar{y}_{\alpha}\right) \rightarrow \infty$ when $\alpha \rightarrow \infty$.

In order to bound $f_{\alpha}$ from above, we mainly use Moser's iteration methods, and it is inequality (30) that allows us to "begin" this iteration.

We recall that $f_{\alpha}$ is a weak solution of

$\left(E_{\alpha}^{\prime}\right) \quad n \mu_{n} \Delta_{n, \bar{g}_{\alpha}} f_{\alpha}+(n-1) n \mu_{n} \frac{\left|\nabla f_{\alpha}\right|_{\bar{g}_{\alpha}}^{n}}{f_{\alpha}}+n \tilde{\varepsilon} k_{\alpha}^{-n} f_{\alpha}^{n-1} \bar{u}_{\alpha}^{n-1}=\lambda_{\alpha} f_{\alpha}^{n}$ 
which precisely means, for any $\phi \in H_{1}^{n}\left(B_{\alpha}\right)$,

$$
n \mu_{n} \int_{B_{\alpha}} \frac{\left|\nabla f_{\alpha}\right|^{n-2} \nabla f_{\alpha} \nabla \phi}{f_{\alpha}^{n-1}} \mathrm{dv}_{\bar{g}_{\alpha}}+n \tilde{\varepsilon} k_{\alpha}^{-n} \int_{B_{\alpha}} \bar{u}_{\alpha}^{n-1} \phi \mathrm{dv}_{\bar{g}_{\alpha}}=\lambda_{\alpha} \int_{B_{\alpha}} f_{\alpha} \phi \mathrm{dv}_{\bar{g}_{\alpha}} .
$$

Testing $\phi=f_{\alpha}^{n-1} \Phi, \Phi \in H_{1}^{n}\left(B_{\alpha}\right)$, we get

$$
\begin{array}{r}
n \mu_{n} \int_{B_{\alpha}}\left|\nabla f_{\alpha}\right|^{n-2} \nabla f_{\alpha} \nabla \Phi \operatorname{dv}_{\bar{g}_{\alpha}}+n(n-1) \mu_{n} \int_{B_{\alpha}} \frac{\left|\nabla f_{\alpha}\right| \bar{g}_{\alpha}}{f_{\alpha}} \Phi \operatorname{dv}_{\bar{g}_{\alpha}} \\
+n \tilde{\varepsilon} k_{\alpha}^{-n} \int_{B_{\alpha}} \bar{u}_{\alpha}^{n-1} f_{\alpha}^{n-1} \Phi \operatorname{dv}_{\bar{g}_{\alpha}}=\lambda_{\alpha} \int_{B_{\alpha}} f_{\alpha}^{n} \Phi \operatorname{dv}_{\bar{g}_{\alpha}} .
\end{array}
$$

In (38) we test $\Phi=\eta^{n} f_{\alpha}^{d}$, where $0<d<1$ and $\eta$ is a cut off function of $B_{\bar{y}_{\alpha}}(R / 2)$. As before, we consider that $\eta$ does not depend on $\alpha$. Considering that $f_{\alpha}>0$, $\bar{u}_{\alpha} \geq 0$ we get

$$
n \mu_{n} \int_{B_{\alpha}}\left|\nabla f_{\alpha}\right|^{n-2} \nabla f_{\alpha} \nabla\left(\eta^{n} f_{\alpha}^{d}\right) \operatorname{dv}_{\bar{g}_{\alpha}} \leq \lambda_{\alpha} \int_{B_{\alpha}} \eta^{n} f_{\alpha}^{n+d} \operatorname{dv}_{\bar{g}_{\alpha}} .
$$

We write $f_{\alpha}^{n+d}=f_{\alpha}^{n \delta^{\prime}} f_{\alpha}$ where $\delta^{\prime}=1+(d-1) / n$. By Hölder's inequality together with (30)

$$
\int_{B_{\alpha}} \eta^{n} f_{\alpha}^{n+d} \operatorname{dv}_{\bar{g}_{\alpha}} \leq C\left(\int_{B_{\alpha}}\left(\eta f_{\alpha}^{\delta^{\prime}}\right)^{n \frac{1+\delta}{\delta}} \mathrm{dv}_{\bar{g}_{\alpha}}\right)^{\frac{\delta}{1+\delta}} .
$$

Hence, for any $\varepsilon_{4}>0$, there exists $C_{\varepsilon_{4}}>0$ such that

$$
\int_{B_{\alpha}} \eta^{n} f_{\alpha}^{n+d} \operatorname{dv}_{\bar{g}_{\alpha}} \leq \varepsilon_{4} \int_{B_{\alpha}}\left|\nabla\left(\eta f_{\alpha}^{\delta^{\prime}}\right)\right|^{n} \operatorname{dv}_{\bar{g}_{\alpha}}+C_{\varepsilon_{4}}\left(\int_{B_{\alpha}} \eta f_{\alpha}^{\delta^{\prime}} \mathrm{dv}_{\bar{g}_{\alpha}}\right)^{n} .
$$

Furthermore,

$$
\begin{array}{r}
\int_{B_{\alpha}}\left|\nabla f_{\alpha}\right|^{n-2} \nabla f_{\alpha} \nabla\left(\eta^{n} f_{\alpha}^{d}\right) \mathrm{dv}_{\bar{g}_{\alpha}} \\
=\int_{B_{\alpha}} d \eta^{n} f_{\alpha}^{d-1}\left|\nabla f_{\alpha}\right| \bar{g}_{\alpha} \mathrm{dv}_{\bar{g}_{\alpha}}+\int_{B_{\alpha}}\left|\nabla f_{\alpha}\right|^{n-2} f_{\alpha}^{d} \nabla \eta^{n} \nabla f_{\alpha} \mathrm{dv}_{\bar{g}_{\alpha}} .
\end{array}
$$

Also

$$
d \int_{B_{\alpha}} \eta^{n} f_{\alpha}^{d-1}\left|\nabla f_{\alpha}\right|_{\bar{g}_{\alpha}}^{n} \mathrm{dv}_{\bar{g}_{\alpha}} \geq C \int_{B_{\alpha}}\left|\nabla\left(\eta f_{\alpha}^{\delta^{\prime}}\right)\right|^{n} \operatorname{dv}_{\bar{g}_{\alpha}}-C \int_{B_{\alpha}} f_{\alpha}^{n \delta^{\prime}}|\nabla \eta|^{n} \mathrm{dv}_{\bar{g}_{\alpha}} .
$$

Putting $f_{\alpha}^{d}=f_{\alpha}^{(d-1) \frac{n-1}{n}} f_{\alpha}^{\frac{\delta^{\prime}}{n}}$ we get for any $\varepsilon_{5}>0$ a $C_{\varepsilon_{5}}>0$ such that

$$
\begin{aligned}
\int_{B_{\alpha}}\left|\nabla f_{\alpha}\right|^{n-2} f_{\alpha}^{d} \nabla \eta^{n} \nabla f_{\alpha} \mathrm{dv}_{\bar{g}_{\alpha}} \geq & -\varepsilon_{5} \int_{B_{\alpha}} \eta^{n} f_{\alpha}^{d-1}\left|\nabla f_{\alpha}\right| \bar{g}_{\bar{g}_{\alpha}} \mathrm{dv}_{\bar{g}_{\alpha}} \\
& -C_{\varepsilon_{5}} \int_{B_{\alpha}}|\nabla \eta|^{n} f_{\alpha}^{n \delta^{\prime}} \mathrm{dv}_{\bar{g}_{\alpha}} .
\end{aligned}
$$

Estimating (41) by means (43) and (42), we get for $\varepsilon_{5}$ sufficiently small

$$
\int_{B_{\alpha}}\left|\nabla f_{\alpha}\right|^{n-2} \nabla f_{\alpha} \nabla\left(\eta^{n} f_{\alpha}^{d}\right) \mathrm{dv}_{\bar{g}_{\alpha}} \geq C \int_{B_{\alpha}}\left|\nabla\left(\eta f_{\alpha}^{\delta^{\prime}}\right)\right|^{n} \mathrm{dv}_{\bar{g}_{\alpha}}-C \int_{B_{\alpha}}|\nabla \eta|^{n} f_{\alpha}^{n \delta^{\prime}} \mathrm{dv}_{\bar{g}_{\alpha}} .
$$

Inserting (39) into (40) and by (44) we finally get for a small $\varepsilon_{4}$

$$
\int_{B_{\alpha}}\left|\nabla\left(\eta f_{\alpha}^{\delta^{\prime}}\right)\right|^{n} \mathrm{dv}_{\bar{g}_{\alpha}} \leq C \int_{B_{\alpha}}|\nabla \eta|^{n} f_{\alpha}^{n \delta^{\prime}} \mathrm{dv}_{\bar{g}_{\alpha}}+C\left(\int_{B_{\alpha}} \eta f_{\alpha}^{\delta^{\prime}} \mathrm{dv}_{\bar{g}_{\alpha}}\right)^{n} .
$$


Choosing $n \delta^{\prime} \leq 1+\delta$ and by (30) we get

$$
\int_{B_{\alpha}}\left|\nabla\left(\eta f_{\alpha}^{\delta^{\prime}}\right)\right|^{n} \mathrm{dv}_{\bar{g}_{\alpha}} \leq C .
$$

Then, for any $p \geq 1$, by (4), (26) (since $\delta^{\prime}<1$ ) and (45)

$$
\begin{aligned}
\left(\int_{B_{\alpha}}\left(\eta f_{\alpha}^{\delta^{\prime}}\right)^{p} \mathrm{dv}_{\bar{g}_{\alpha}}\right)^{\frac{n}{p}} & \leq \varepsilon \int_{B_{\alpha}}\left|\nabla\left(\eta f_{\alpha}^{\delta^{\prime}}\right)\right|^{n} \mathrm{dv}_{\bar{g}_{\alpha}}+C_{\varepsilon}\left(\int_{B_{\alpha}} \eta f_{\alpha}^{\delta^{\prime}} \mathrm{dv}_{\bar{g}_{\alpha}}\right)^{n} \\
& \leq C .
\end{aligned}
$$

Therefore, for $q$ arbitrarily large

$$
\int_{B_{\bar{y}_{\alpha}}(R / 2)} f_{\alpha}^{q} \mathrm{dv}_{\bar{g}_{\alpha}} \leq C .
$$

As shown by Serrin [20] (see also [22] or [23]) we deduce that

$$
\lim _{\alpha \rightarrow+\infty}\left\|f_{\alpha}\right\|_{L^{\infty}\left(B_{\bar{y}_{\alpha}}(R / 2)\right)} \leq C .
$$

This contradicts (25).

Step 8. We now prove that inequality (23) implies

$$
\int_{B_{x_{\alpha}}(\delta)}\left|\nabla u_{\alpha}\right|^{n} \mathrm{~d}_{g}^{2}\left(x, x_{\alpha}\right) \mathrm{dv}_{g} \leq C .
$$

We set $r_{\alpha}=\mathrm{d}_{g}\left(x, x_{\alpha}\right)$. Since $r_{\alpha}^{2}$ is a Lipschitzian function on $M$, we have $r_{\alpha}^{2} u_{\alpha} \in$ $H_{1}^{n}$. In (7) we test $\Phi=r_{\alpha}^{2} u_{\alpha}$ and we obtain

$$
n \mu_{n} \int_{M}\left|\nabla u_{\alpha}\right|^{n-2} \nabla u_{\alpha} \nabla\left(r_{\alpha}^{2} u_{\alpha}\right) \mathrm{dv}_{g} \leq \lambda_{\alpha} \frac{\int_{M} e^{u_{\alpha}} u_{\alpha} r_{\alpha}^{2} \mathrm{dv}_{g}}{\int_{M} e^{u_{\alpha}} \mathrm{dv}_{g}} .
$$

For any $\alpha,\left|\nabla r_{\alpha}\right| \leq C$, we get

$$
n \mu_{n} \int_{M} r_{\alpha}^{2}\left|\nabla u_{\alpha}\right|^{n} \operatorname{dv}_{g} \leq \lambda_{\alpha} \frac{\int_{M} e^{u_{\alpha}} u_{\alpha} r_{\alpha}^{2} \mathrm{dv}_{g}}{\int_{M} e^{u_{\alpha}} \mathrm{dv}_{g}}+C \int_{M}\left|\nabla u_{\alpha}\right|^{n-1} u_{\alpha} \mathrm{dv}_{g} .
$$

Choose $n-1<p<n$ and applying Hölder's inequality,

$$
\int_{M}\left|\nabla u_{\alpha}\right|^{n-1} u_{\alpha} \mathrm{dv}_{g} \leq\left(\int_{M}\left|\nabla u_{\alpha}\right|^{p} \mathrm{dv}_{g}\right)^{\frac{n-1}{p}}\left(\int_{M} u_{\alpha}^{\frac{p}{p-n+1}} \mathrm{dv}_{g}\right)^{\frac{p-n+1}{p}} .
$$

By (12) we have $\int_{M}\left|\nabla u_{\alpha}\right|^{p} \operatorname{dv}_{g} \leq C$ for any $p<n$ and by (10) $\int_{M} u_{\alpha}^{p /(p-n+1)} \operatorname{dv}_{g} \leq$ $C$ for any $p>n-1$. Therefore

$$
n \mu_{n} \int_{M} r_{\alpha}^{2}\left|\nabla u_{\alpha}\right|^{n} \mathrm{dv}_{g} \leq \lambda_{\alpha} \frac{\int_{M} e^{u_{\alpha}} u_{\alpha} r_{\alpha}^{2} \mathrm{dv}_{g}}{\int_{M} e^{u_{\alpha}} \mathrm{dv}_{g}}+C .
$$

Furthermore

$$
\int_{M} e^{u_{\alpha}} u_{\alpha} r_{\alpha}^{2} \mathrm{dv}_{g} \leq\left(\int_{M} e^{u_{\alpha}} r_{\alpha}^{n} u_{\alpha}^{n / 2} \mathrm{dv}_{g}\right)^{2 / n}\left(\int_{M} e^{u_{\alpha}} \mathrm{dv}_{g}\right)^{\frac{n-2}{n}} .
$$

This implies

$$
\int_{M} r_{\alpha}^{2}\left|\nabla u_{\alpha}\right|^{n} \mathrm{dv}_{g} \leq C\left(\frac{\int_{M} e^{u_{\alpha}} r_{\alpha}^{n} u_{\alpha}^{n / 2} \mathrm{dv}_{g}}{\int_{M} e^{u_{\alpha}} \mathrm{dv}_{g}}\right)^{2 / n}+C
$$


and by (23) and (10)

$$
\int_{M} r_{\alpha}^{2}\left|\nabla u_{\alpha}\right|^{n} \mathrm{dv}_{g} \leq C\left(\int_{M} u_{\alpha}^{n / 2} \mathrm{dv}_{g}\right)^{2 / n}+C \leq C .
$$

Step 9. We now get to our final argument.

We extend the metric $g$ in $x_{\alpha}$ and we get

$$
\left(1-C r_{\alpha}^{2}\right) \mathrm{dv}_{g} \leq \mathrm{d} \xi \leq\left(1+C r_{\alpha}^{2}\right) \mathrm{dv}_{g}
$$

where $C$ does not depend on $\alpha, r_{\alpha}=\mathrm{d}_{g}\left(x, x_{\alpha}\right)$, $\mathrm{d} \xi$ the Euclidean element of volume. We also have, for any $v \in H_{1}^{n}(B)$, where $B$ is a ball of center $x_{\alpha}$

$$
|\nabla v|_{\xi}^{n} \leq|\nabla v|_{g}^{n}\left(1+C r_{\alpha}^{2}\right)
$$

Hence, for any $v \in H_{1}^{n}(B)$

$$
\int_{B} e^{v} \mathrm{dv}_{g} \leq \int_{B} e^{v} \mathrm{~d} \xi+C \int_{B} e^{v} r_{\alpha}^{2} \mathrm{dv}_{g}
$$

and by $(5)$

$$
\begin{aligned}
\int_{B} e^{v} \mathrm{dv}_{g} & \leq C \exp \left(\mu_{n} \int_{B}|\nabla v|_{\xi}^{n} \mathrm{~d} \xi\right)+C \int_{B} e^{v} r_{\alpha}^{2} \mathrm{dv}_{g} \\
& \leq C \exp \left(\mu_{n} \int_{B}|\nabla v|_{g}^{n} \mathrm{dv}_{g}+C \int_{B}|\nabla v|_{g}^{n} r_{\alpha}^{2} \mathrm{dv}_{g}\right)+C \delta^{2} \int_{B} e^{v} \mathrm{dv}_{g} .
\end{aligned}
$$

For $\delta$ small

$$
\int_{B} e^{v} \mathrm{dv}_{g} \leq C \exp \left(\mu_{n} \int_{B}|\nabla v|_{g}^{n} \mathrm{dv}_{g}\right) \exp \left(\int_{B}|\nabla v|_{g}^{n} r_{\alpha}^{2} \mathrm{dv}_{g}\right) .
$$

Hence, with $\eta$ a cut off function of $B_{x_{\alpha}}(\delta), \eta=1$ on $B_{x_{\alpha}}(\delta), \eta=0$ on $M \backslash B_{x_{\alpha}}(2 \delta)$, we get by (46)

$$
\int_{B_{x_{\alpha}}(\delta)} e^{\eta u_{\alpha}} \mathrm{dv}_{g} \leq C \exp \left(\mu_{n} \int_{B_{x_{\alpha}}(\delta)}\left|\nabla\left(\eta u_{\alpha}\right)\right|_{g}^{n} \mathrm{dv}_{g}\right)+C .
$$

This inequality is sufficient to get the contradiction, as shown in [15]. For completeness, we reproduce it here.

By definition of a concentration point

$$
\varlimsup_{\alpha \rightarrow+\infty} \frac{\int_{B_{x_{\alpha}}(\delta / 2)} e^{u_{\alpha}} \mathrm{dv}_{g}}{\int_{M} e^{u_{\alpha}} \mathrm{dv}_{g}}>0,
$$

hence

$$
\varlimsup_{\alpha \rightarrow+\infty} \frac{\int_{M \backslash B_{x_{\alpha}}(\delta / 2)} e^{u_{\alpha}} \mathrm{dv}_{g}}{\int_{M} e^{u_{\alpha}} \mathrm{dv}_{g}}=k<1,
$$

and in view of (14), this implies that

$$
\int_{M \backslash B_{x_{\alpha}}(\delta)}\left|\nabla u_{\alpha}\right|^{n} \leq C .
$$


We then obtain

$$
\begin{aligned}
& \int_{M} e^{u_{\alpha}} \mathrm{dv}_{g}=\int_{M \backslash B_{x_{\alpha}}(\delta)} e^{u_{\alpha}} \mathrm{dv}_{g}+\int_{B_{x_{\alpha}}(\delta)} e^{u_{\alpha}} \mathrm{dv}_{g} \\
& \leq \int_{M \backslash B_{x_{\alpha}}(\delta)} e^{u_{\alpha}} \mathrm{dv}_{g}+\int_{B_{x_{\alpha}}(\delta)} e^{\eta u_{\alpha}} \mathrm{dv}_{g} \\
& \leq C \exp \left(C \int_{M \backslash B_{x_{\alpha}}(\delta)}\left|\nabla u_{\alpha}\right|^{n} \mathrm{dv}_{g}+C \int_{M \backslash B_{x_{\alpha}}(\delta)} u_{\alpha}^{n} \mathrm{dv}_{g}\right) \\
& +C \exp \left(\mu_{n} \int_{B_{x_{\alpha}}(\delta)}\left|\nabla\left(\eta u_{\alpha}\right)\right|^{n} \mathrm{dv}_{g}\right) .
\end{aligned}
$$

The last inequality follows from (47). Since $\int_{M \backslash B_{x_{\alpha}}(\delta)}\left|\nabla u_{\alpha}\right|^{n} \mathrm{dv}_{g} \leq C$ and

$$
\int_{M \backslash B_{x_{\alpha}}(\delta)} u_{\alpha}^{n} \mathrm{dv}_{g} \leq C
$$

this leads to

$$
\begin{aligned}
& \int_{M} e^{u_{\alpha}} \mathrm{dv}_{g} \leq C \exp \left(\mu_{n} \int_{B_{x_{\alpha}}(\delta)}\left|\nabla\left(\eta u_{\alpha}\right)\right|^{n} \mathrm{dv}_{g}\right)+C \\
& \leq C \exp \left(\mu_{n} \int_{B_{x_{\alpha}}(\delta)}\left|\nabla u_{\alpha}\right|^{n} \mathrm{dv}_{g}+C \int_{M} u_{\alpha}^{n} \mathrm{dv}_{g}+C \int_{M \backslash B_{x_{\alpha}}(\delta)}\left|\nabla u_{\alpha}\right|^{n} \mathrm{dv}_{g}\right) .
\end{aligned}
$$

With our initial assumption

$$
\mu_{n} \int_{M}\left|\nabla u_{\alpha}\right|^{n} \mathrm{dv}_{g}+\tilde{\varepsilon} \int_{M} u_{\alpha}^{n} \mathrm{dv}_{g}+\alpha \leq \mu_{n} \int_{B}\left|\nabla u_{\alpha}\right|^{n} \mathrm{dv}_{g}+C,
$$

which is absurd since $\alpha \rightarrow+\infty$. The proposition is proven.

\section{Proof of the Theorem}

Once again, we proceed by contradiction. Assume that for any $\alpha>0$, there exists $v_{\alpha} \in H_{1}^{n}$ satisfying

$$
\ln \left(\int_{M} e^{v_{\alpha}} \mathrm{dv}_{g}\right)>\alpha+\mu_{n} \int_{M}\left|\nabla v_{\alpha}\right|^{n} \mathrm{dv}_{g}
$$

Let $H=\left\{u \in H_{1}^{n} \mid \int_{M} e^{u} \mathrm{dv}_{g}>1, \int_{M} u \mathrm{dv}_{g}=0\right\}$. We consider the functional $J_{\alpha}$ such that for any $u \in H$

$$
J_{\alpha}(u)=\frac{\alpha+\mu_{n} \int_{M}|\nabla u|^{n} \mathrm{dv}_{g}}{\ln \left(\int_{M} e^{u} \mathrm{dv}_{g}\right)} .
$$

Let $\nu_{\alpha}=\inf _{u \in H} J_{\alpha}(u)$. With our initial assumption, we have that $0<\nu_{\alpha}<1$. Standard variational techniques give us for any $\alpha$ the existence of $u_{\alpha} \in H$ such that $J_{\alpha}\left(u_{\alpha}\right)=\nu_{\alpha}$, i.e.

$$
\nu_{\alpha}=\frac{\alpha+\mu_{n} \int_{M}\left|\nabla u_{\alpha}\right|^{n} \mathrm{dv}_{g}}{\ln \left(\int_{M} e^{u_{\alpha}} \operatorname{dv}_{g}\right)} .
$$


We also get, for any $\phi \in H_{1}^{n}, J_{\alpha, u_{\alpha}}^{\prime}(\phi)=\gamma_{\alpha} \int_{M} \phi \mathrm{dv}_{g}$, which means, given the expression of $J_{\alpha, u_{\alpha}}^{\prime}$ : for any $\phi \in H_{1}^{n}$

$$
n \mu_{n} \int_{M}\left|\nabla u_{\alpha}\right|^{n-2} \nabla u_{\alpha} \nabla \phi \mathrm{dv}_{g}-\nu_{\alpha} \frac{\int_{M} e^{u_{\alpha}} \phi \mathrm{dv}_{g}}{\int_{M} e^{u_{\alpha}} \mathrm{dv}_{g}}+\frac{\nu_{\alpha}}{\operatorname{vol}(M)} \int_{M} \phi \mathrm{dv}_{g}=0 .
$$

We deduce from the Proposition that there exists $\varepsilon, C_{\varepsilon}$ such that, for any $u \in H_{1}^{n}$

$$
\ln \left(\int_{M} e^{u} \mathrm{dv}_{g}\right) \leq C_{\varepsilon}+\mu_{n} \int_{M}|\nabla u|^{n} \mathrm{dv}_{g}+\varepsilon \int_{M}|u|^{n} \mathrm{dv}_{g} .
$$

According to (48) and (50), we only have to prove that there exists $C$ (not depending on $\alpha$ ) such that $\int_{M}\left|u_{\alpha}\right|^{n} \mathrm{dv}_{g} \leq C$ to get a contradiction. Indeed,

$$
\alpha+\mu_{n} \int_{M}\left|\nabla u_{\alpha}\right|^{n} \mathrm{dv}_{g} \leq C_{\varepsilon}+\mu_{n} \int_{M}\left|\nabla u_{\alpha}\right|^{n} \mathrm{dv}_{g}+\varepsilon \int_{M}\left|u_{\alpha}\right|^{n} \mathrm{dv}_{g}
$$

implies

$$
\alpha \leq C_{\varepsilon}+\varepsilon \int_{M}\left|u_{\alpha}\right|^{n} \mathrm{dv}_{g} .
$$

To estimate $\int_{M}\left|u_{\alpha}\right|^{n} \mathrm{dv}_{g}$, we test in (49) a certain $\phi=f \circ u_{\alpha}$ defined as follows. Let

$$
\begin{aligned}
\Omega_{1} & =\left\{x \in M \mid u_{\alpha}(x) \geq 1\right\}, \\
\Omega_{-1} & =\left\{x \in M \mid u_{\alpha}(x) \leq-1\right\}, \\
\Omega & =\left\{x \in M|| u_{\alpha}(x) \mid \leq 1\right\}
\end{aligned}
$$

$\left(\Omega_{1}, \Omega_{-1}, \Omega\right.$ depending on $\left.\alpha\right)$, and let $f$ the real function defined by

$$
\begin{aligned}
f(x) & =x^{-1 / n} \text { when } x \geq 1 \\
& =x \text { when }-1 \leq x \leq 1 \\
& =-|x|^{-1 / n} \text { when } x \leq-1 .
\end{aligned}
$$

The function $f$ is Lipschitzian and therefore $\phi=f \circ u_{\alpha} \in H_{1}^{n}$. We get

$$
\int_{M}\left|\nabla u_{\alpha}\right|^{n-2} \nabla u_{\alpha} \nabla \phi \mathrm{dv}_{g}=-\frac{1}{n} \int_{\Omega_{1} \cup \Omega_{-1}}\left|u_{\alpha}\right|^{-(n+1) / n}\left|\nabla u_{\alpha}\right|^{n} \mathrm{dv}_{g}+\int_{\Omega}\left|\nabla u_{\alpha}\right|^{n} \mathrm{dv}_{g} \text {. }
$$

Testing $\phi$ in (49), we get

$$
\begin{aligned}
-\mu_{n} & \int_{\Omega_{1} \cup \Omega_{-1}}\left|u_{\alpha}\right|^{-(n+1) / n}\left|\nabla u_{\alpha}\right|^{n} \operatorname{dv}_{g}+n \mu_{n} \int_{\Omega}\left|\nabla u_{\alpha}\right|^{n} \mathrm{dv}_{g} \\
& +\frac{\nu_{\alpha}}{\operatorname{vol}(M)} \int_{\Omega_{1}}\left|u_{\alpha}\right|^{-1 / n} \operatorname{dv}_{g}-\frac{\nu_{\alpha}}{\operatorname{vol}(M)} \int_{\Omega_{-1}}\left|u_{\alpha}\right|^{-1 / n} \operatorname{dv}_{g}+\frac{\nu_{\alpha}}{\operatorname{vol}(M)} \int_{\Omega} u_{\alpha} \mathrm{dv}_{g} \\
(51) & =\nu_{\alpha} \frac{\int_{M} e^{u_{\alpha}} \Phi \operatorname{dv}_{g}}{\int_{M} e^{u_{\alpha}} \operatorname{dv}_{g}} .
\end{aligned}
$$

By construction of $\phi$,

$$
\frac{\int_{M} e^{u_{\alpha}} \phi \mathrm{dv}_{g}}{\int_{M} e^{u_{\alpha}} \mathrm{dv}_{g}} \leq C
$$

and $\int_{M} \phi \mathrm{dv}_{g} \leq C$. By (51), this leads to

$$
n \mu_{n} \int_{M}\left|\nabla u_{\alpha}\right|^{n-2} \nabla u_{\alpha} \nabla \phi \operatorname{dv}_{g} \leq C .
$$


The key idea now is to consider the function $v$ such that $|v| \leq 1$ on $M$ defined by

$$
\begin{aligned}
v & =u_{\alpha}^{\left(n^{2}-n-1\right) / n^{2}} \text { on } \Omega_{1} \\
& =u_{\alpha} \text { on } \Omega \\
& =-\left|u_{\alpha}\right|^{\left(n^{2}-n-1\right) / n^{2}} \text { on } \Omega_{-1} .
\end{aligned}
$$

In order to go on with the proof, we need the following Lemma.

Lemma. Let $(M, g)$ be a Riemannian $n$-manifold. For any $p \geq 1, q>0$, there exists $C>0$ such that, for any $u \in H_{1}^{n}(M)$,

$$
\left[\int_{M}|u|^{p} \mathrm{dv}_{g}\right]^{n / p} \leq C \int_{M}|\nabla u|^{n} \mathrm{dv}_{g}+C\left|\int_{M} h(u) \mathrm{dv}_{g}\right|^{q},
$$

where $h$ is the real function defined by $h(x)=(\operatorname{sign}(x))|x|^{n / q}$.

Proof. We consider the functional $L$

$$
L(u)=\frac{\int_{M}|\nabla u|^{n} \mathrm{dv}_{g}+\left|\int_{M} h(u) \mathrm{dv}_{g}\right|^{q}}{\left[\int_{M}|u|^{p} \mathrm{dv}_{g}\right]^{n / p}}, u \in H_{1}^{n} .
$$

Since $L$ is homogeneous in $u$, we consider $P=\left\{\left.u \in H_{1}^{n}\left|\int_{M}\right| u\right|^{p} \operatorname{dv}_{g}=1\right\}$ and $\mu=\inf _{u \in P} L(u)$. Using the fact that for any $k \geq 1$, the embedding of $H_{1}^{n}$ in $L^{k}$ is compact, standard techniques give the existence of $w \in P$ such that $L(w)=\mu$. It is clear that $\mu \geq 0$; we now want to prove that $\mu>0$. If $\mu=0$, since $L(w)=\mu$, then $\int_{M}|\nabla w| \mathrm{dv}_{g}=0$ and $w$ is constant. Since $\int_{M} h(w) \mathrm{dv}_{g}=0$ then $w \equiv 0$. But $\int_{M}|w|^{p} \mathrm{dv}_{g}=1$ since $v$ is in $P$, which is absurd. This way $\mu>0$, and we proved (53).

Proof. We continue with the proof of the Theorem. We apply (53) to $v$ defined above with $q=\left(n^{2}-n-1\right) / n$. For any $p \geq 1$

$$
\begin{gathered}
{\left[\int_{\Omega_{1}} u_{\alpha}^{p\left(n^{2}-n-1\right) / n^{2}} \mathrm{dv}_{g}-\int_{\Omega_{-1}}\left|u_{\alpha}\right|^{p\left(n^{2}-n-1\right) / n^{2}} \mathrm{dv}_{g}+\int_{\Omega} u_{\alpha}^{p} \mathrm{dv}_{g}\right]^{n / p}} \\
\quad \leq C \int_{M}|\nabla v|^{n} \mathrm{dv}_{g}+C\left(\int_{\Omega_{1} \cup \Omega_{-1}} u_{\alpha} \mathrm{dv}_{g}+\int_{\Omega} h\left(u_{\alpha}\right) \mathrm{dv}_{g}\right)^{\left(n^{2}-n-1\right) / n},
\end{gathered}
$$

where $h\left(u_{\alpha}\right)=\left(\operatorname{sign}\left(u_{\alpha}\right)\right)\left|u_{\alpha}\right|^{n^{2} /\left(n^{2}-n-1\right)}$. We verify that

$$
\int_{M}|\nabla v|^{n} \mathrm{dv}_{g} \leq C \int_{M}\left|\nabla u_{\alpha}\right|^{n-2} \nabla u_{\alpha} \nabla \phi \mathrm{dv}_{g}
$$

where $\phi=f \circ u_{\alpha}$, and by (52), $\int_{M}|\nabla v|^{n} \operatorname{dv}_{g} \leq C$. Since $M=\Omega_{1} \cup \Omega \cup \Omega_{-1}$,

$$
\int_{\Omega_{1} \cup \Omega_{-1}} u_{\alpha} \mathrm{dv}_{g}+\int_{\Omega} h\left(u_{\alpha}\right) \mathrm{dv}_{g}=\int_{M} u_{\alpha} \mathrm{dv}_{g}-\int_{\Omega} u_{\alpha} \mathrm{dv}_{g}+\int_{\Omega} h\left(u_{\alpha}\right) \mathrm{dv}_{g} .
$$

Since $u_{\alpha} \in H, \int_{M} u_{\alpha} \mathrm{dv}_{g}=0$, and since on $\Omega,\left|u_{\alpha}\right| \leq 1$ we get $\int_{\Omega} h\left(u_{\alpha}\right) \mathrm{dv}_{g} \leq C$ and finally

$$
\left[\int_{\Omega_{1}} u_{\alpha}^{p\left(n^{2}-n-1\right) / n^{2}} \mathrm{dv}_{g}-\int_{\Omega_{-1}}\left|u_{\alpha}\right|^{p\left(n^{2}-n-1\right) / n^{2}} \mathrm{dv}_{g}+\int_{\Omega} u_{\alpha}^{p} \mathrm{dv}_{g}\right]^{n / p} \leq C .
$$


With $p=n^{3} /\left(n^{2}-n-1\right)$ we get

$$
\int_{M} u_{\alpha}^{n} \mathrm{dv}_{g}-\int_{\Omega} u_{\alpha}^{n} \mathrm{dv}_{g}+\int_{\Omega} u_{\alpha}^{n^{3} /\left(n^{2}-n-1\right)} \mathrm{dv}_{g} \leq C .
$$

Using once again the fact that on $\Omega,\left|u_{\alpha}\right| \leq 1$, we get that $\int_{M} u_{\alpha}^{n} \mathrm{dv}_{g} \leq C$, which contradicts $(\star)$. The theorem is proved.

\section{REFERENCES}

[1] Aubin, Th.: "Espaces de Sobolev sur les variétés Riemanniennes", Bulletin des Sciences Mathématiques, 100 (1976), 149-173. MR0488125 (58:7692)

[2] Aubin, Th.: "Meilleures constantes dans le théorème d'inclusion de Sobolev et un théorème de Fredholm non linéaire pour la transformation conforme de la courbure scalaire", Journ. of Funct. Anal. 32 (1979), 148-174. MR534672 (80i:58043)

[3] Aubin, Th.: Some non linear problems in Riemannian geometry, Springer Monographs in Mathematics, 1998.

[4] Aubin, Th. and Cotsiolis, A.: "Equations elliptiques non linéaires sur $S_{n}$ dans le cas supercritique", Bulletin des Sciences Mathématiques 123, 1999, 33-45. MR1672546 (2000d:35048)

[5] Bandle, C.: "Green's function, harmonic transplantation, and best Sobolev constant in spaces of constant curvature", Trans. Amer. Math. Soc. 350, 1998, 1103-1128. MR1458294 (98g:35045)

[6] Bandle, C. and Peletier, L.A.: "Best Sobolev constants and Emden equations for the critical exponent in $S^{3}$ ", Math. Ann. 313, 1999, 83-93. MR1666821 (2001c:35030)

[7] Bandle, C. "Sobolev inequalities and quasilinear elliptic boundary value problems" Basler preprint, 2001.

[8] Cherrier, P. "Une inégalité de Sobolev sur les variétés Riemanniennes", Bulletin des Sciences Mathématiques 103, 1979, 353-374. MR548913 (81a:58055)

[9] Z. Djadli, and O. Druet, Extremal functions for optimal Sobolev inequalities on compact manifolds. Calc. Var. 12 (2001), 59-84. MR1808107 (2002d:58042)

[10] Druet, O.: "The best constants problem in Sobolev inequalities" Mathematische Annalen 314, 1999, 327-346. MR1697448 (2000d:58033)

[11] Druet, O.: "Generalized scalar curvature type equations on compact Riemannian manifolds", Proceedings of the Royal Society of Edinburgh 130A, 2000, 767-788. MR1776675 (2001g:53070)

[12] Druet, O. and Robert, F.: "Asymptotic profile for the sub-extremals of the sharp Sobolev inequalities on the sphere", Communications in Partial Differential Equations 26, 2001, 743778. MR1843283 (2002k:58045)

[13] Faget, Z.: "Best constants in Sobolev inequalitites on Riemannian manifolds in the presence of symmetries" Potential Analysis 17 (2002), 105-124. MR1908673 (2003g:58054)

[14] Faget, Z. : "Optimal constants in critical Sobolev inequalities on Riemannian manifolds in the presence of symmetries", Annals of Global Analysis and Geometry 24, (2003), 161-200. MR1990113 (2004d:58028)

[15] Faget, Z. "Best constant in the exceptional case of Sobolev inequalities", Math. Zeit. 252, 2006, 133-146. MR2209155 (2007c:58030)

[16] Hebey, E.: Sobolev spaces on Riemannian manifolds, Lecture Notes in Mathematics, 1635, Springer, Berlin. MR1481970 (98k:46049)

[17] Hebey, E.: Non linear analysis on manifolds: Sobolev spaces and inequalities, Courant Institute of Mathematical Sciences, Lecture Notes in Mathematics 5, 1999. MR1688256 (2000e:58011)

[18] Hebey, E. and Vaugon, M.: "Sobolev spaces in the presence of symmetries", Journal de Mathématiques Pures et Appliquées 76, 1997, 859-881. MR1489942 (98m:46047)

[19] Hebey, E. and Vaugon, M.: "Meilleures constantes dans le théorème d'inclusion de Sobolev", Annales de l'Institut Henri Poincaré, Analyse non-linéaire 13, 1996, 57-93. MR1373472 (96m:46054)

[20] Serrin, J.: "Local behavior of solutions of quasilinear equations", Acta Mathematica 111, 1964, 247-302. MR0170096 (30:337)

[21] Tolksdorf, P.: "Regularity results for a more general class of quasilinear elliptic equations", Journal of differential Equations 51, 1984, 126-150. MR727034 (85g:35047) 
[22] Trudinger, N.: "On Harnack type inequalities and their applications to quasilinear elliptic equations", Communications on Pure and Applied Mathematics 20, 1967, 721-747. MR0226198 (37:1788)

[23] Véron, L.: Singularities of solutions of second order quasilinear equations, Pitman Research Notes in Mathematics Series, 353, Longman, 1996. MR1424468 (98b:35053)

Departement Mathematik, ETH-Zentrum, CH-8092, Zurich, Switzerland

E-mail address: zoe.faget@math.ethz.ch

Current address: Equipe Géométrie et Dynamique, Institut Mathématiques, 173 rue de Chevaleret, 75013 Paris, France

E-mail address: fagetzoe@math.jussieu.fr 